\title{
Construir el conocimiento en la era digital: retos y reflexiones
}

\section{Building knowledge in the digital age: challenges and reflections}

\author{
Marta Marimon-Martí \\ Universitat de Vic-Universitat Central de Catalunya. Vic, España \\ marta.marimon@uvic.cat \\ Julio Cabero \\ Universidad de Sevilla. Sevilla, España \\ cabero@us.es \\ Linda Castañeda \\ Universidad de Murcia. Murcia, España \\ lindacq@um.es \\ César Coll \\ Universitat de Barcelona. Barcelona, España \\ ccoll@ub.edu \\ Janaina Minelli de Oliveira \\ Universitat Rovira i Virgili. Tarragona, España \\ Janaina.oliveira@urv.cat \\ Maria Jesús Rodríguez-Triana \\ Tallinn University. Tallin, Estonia \\ mjrt@tlu.ee
}

\section{Resumen}

Este trabajo identifica cinco grandes retos que enfrentan los modelos emergentes de construcción y creación de conocimiento en contextos digitales: la democratización de la educación y la toma en consideración de las cuestiones relacionadas con la diversidad en un sentido amplio; el impacto de la pandemia de la COVID-19 y sus implicaciones en lo que concierne a los usos de las TIC en la educación; la existencia de múltiples y diversos contextos de construcción y creación de conocimiento, su interrelación y su necesaria articulación; la curación de contenidos, su impacto en las prácticas educativas y sus implicaciones para la formación del profesorado y para el aprendizaje del alumnado; y las analíticas de aprendizaje y su papel en la toma de decisiones orientadas a mejorar la eficacia de la enseñanza y optimizar el aprendizaje. Estos cinco ámbitos son revisados así como sus aspectos más significativos, que deberían ser objeto de especial atención en la investigación y la innovación educativa en los próximos años. Se incluye, además, un anexo dedicado a presentar un glosario de algunos términos utilizados en el trabajo.

Palabras clave: Analíticas de aprendizaje, contextos de aprendizaje, creación de conocimiento, curación de contenidos, democratización, tecnología educativa.

\begin{abstract}
This work identifies five major challenges faced by emerging models of construction and creation of knowledge in digital contexts: the democratization of education and the analysis of issues related to diversity in a broad sense; the impact of the COVID19 pandemic and its implications regarding the uses of ICT in education; the existence of multiple and diverse contexts for the construction and creation of knowledge, their interrelation and their necessary articulation; content curation, its impact on educational practices, its implications for teacher training and for student learning; and learning analytics and their role in decision-making aimed at improving teaching effectiveness and optimizing learning. These five areas are reviewed as well as their most significant aspects, which should be the object of special attention in
\end{abstract}


research and educational innovation in the coming years. It also includes an annex dedicated to presenting a glossary of some terms used in the work.

Key words: Learning analytics, learning contexts, knowledge creation, content curation, democratization, educational technology.

\section{Introducción: del FIET 2014 al FIET 2021}

Este trabajo presenta una serie de reflexiones a modo de ensayo científico dedicado a los nuevos modelos de construcción del conocimiento en la era digital. Estas reflexiones son el resultado de la discusión entre un grupo de expertos en este ámbito en el marco del Fórum Internacional de Educación y Tecnología (FIET) celebrado en el 2021. Se trata de un texto original e inédito con entidad propia en clave de ensayo, que parte de un proceso de debate iniciado en la primera edición del FIET celebrada en 2014 que se contextualiza en las siguientes líneas, y que se ha retomado en esta nueva edición para valorar lo que ha transcurrido durante este período en relación a esta problemática. El resultado del proceso de discusión que se presenta en este trabajo pretende contribuir a una mejor comprensión de algunos temas claves relacionados con el diseño de las prácticas educativas en la era digital, y de cómo estos diseños configuran modelos emergentes de producción del conocimiento. Nuestro objetivo es que las ideas generadas a partir de este debate interno permitan identificar una serie de retos que, a nuestro entender, deberían ser abordados por la investigación y la innovación educativa futuras.

Como hemos mencionado, este proceso se inició en 2014, cuando un grupo de expertos de ámbito nacional e internacional llevó a cabo un primer proceso de reflexión sobre estas cuestiones que presentamos a continuación (de Oliveira et al., 2015; Marimon et al., 2016). Por un lado, el subgrupo de expertos de ámbito nacional identificó cuatro factores claves de estos nuevos modelos emergentes de producción de conocimiento en términos de potencialidades y necesidades:

1. El aprendizaje a lo largo y ancho de la vida, para una formación de aprendices competentes, que deben adquirir los saberes fundamentales y las habilidades del siglo XXI que les permitan aprender a aprender.

2. El aprendizaje mediado y modelado por las tecnologías, con multiplicidad de lenguajes y formatos de representación, que supone avanzar hacia una alfabetización que permita a los aprendices participar eficazmente y colaborativamente en las prácticas sociales y culturales de un mundo digital.

3. El aprendizaje sin barreras, que incorpore una visión amplia de la educación y su articulación con otros escenarios y agentes educativos para dar continuidad entre los aprendizajes formales e informales como fuente de acceso al conocimiento.

4. La personalización del aprendizaje, que implica una resignificación de las tareas de enseñar y aprender, incorporando las trayectorias individuales de aprendizaje, el interés por aprender y la identidad del aprendiz al foco de la acción educativa.

Por otra parte, el subgrupo de expertos de ámbito internacional sugirió que es esencial promover el desarrollo de nuevos conocimientos sobre la dinámica de la construcción del conocimiento y las consecuencias que estos cambios tienen sobre el papel de los docentes y los propósitos educativos en el contexto comunicativo del siglo XXI. Estos nuevos conocimientos fueron identificados a partir de tres categorías que se entrelazan e interconectan entre sí, especialmente cuando consideramos las especificidades del diseño 
y los problemas complejos del mundo real: el conocimiento fundacional (lo que necesitamos saber), el conocimiento meta o metaconocimiento (como actuamos sobre este conocimiento) y el conocimiento humanístico (los valores que aportamos a nuestro conocimiento y acción).

La discusión en torno a estas consideraciones y la reflexión conjunta entre los dos subgrupos de expertos permitió identificar cinco grandes temas o ideas que nutren los modelos emergentes de construcción del conocimiento en la era digital:

1. La aparición de nuevos modelos de construcción del conocimiento basados en teorías emergentes que no se centran exclusivamente en la educación formal, sino que proponen una nueva ecología del aprendizaje fundamentada en la ubicuidad de las experiencias de aprendizaje que favorecen las tecnologías digitales.

2. Estos nuevos modelos de construcción del conocimiento están situados y contextualizados dentro de las interacciones sociales que vinculan el individuo, el grupo y la comunidad en general mediante diferentes niveles de solidaridad referidos a las dinámicas de producción, consumo y distribución del conocimiento, rompiendo barreras geográficas, temporales e incluso culturales.

3. Estos condicionantes permiten la aparición de pedagogías de abajo hacia arriba, en las que los profesionales (maestros, profesores, educadores, entre otros) buscan maneras de responder adecuadamente a las nuevas necesidades que se les plantean a través del diálogo y de la participación en comunidades de práctica a través de las redes sociales.

4. Los enfoques multinivel y de abajo hacia arriba en la construcción del conocimiento y con el uso de las tecnologías tienen también el potencial creativo necesario para romper fronteras mediante el desarrollo de nuevas posibilidades que van más allá de los límites de las estructuras pedagógicas tradicionales, priorizando así los intereses del alumnado, y devolviendo la alegría al propio proceso de aprendizaje.

5. Las nuevas posibilidades de las redes digitales y globales permiten la licuefacción de las identidades étnico-culturales de los estudiantes en formas que antes no eran posibles, mediante su participación en comunidades de aprendizaje en línea en las que pueden explorar, expresar y transformar sus identidades personales, profesionales y culturales.

Estas no son, sin embargo, las únicas cuestiones importantes en las que pensar, sino solo ideas que centraron el debate en el 2014 por considerar que englobaban algunos de los temas que los profesionales de la educación deben tener presentes al mirar hacia el futuro. Partiendo de estas reflexiones iniciales y situados en el momento actual, es lógico plantearse algunas preguntas respecto a las conclusiones de la reflexión realizada años atrás: ¿los retos que intuíamos en 2014 han sido afrontados y superados?, ¿hemos avanzado hacia nuevos modelos de construcción del conocimiento sensibles con los temas señalados?, ¿cuáles son los nuevos retos a los que nos enfrentamos hoy? La actual situación provocada por la pandemia del Covid-19 ha comportado cambios en las modalidades de enseñanza y aprendizaje que han hecho aflorar las posibilidades y oportunidades que ofrecen los entornos de formación híbridos. En este contexto, ¿qué nuevas consideraciones debemos tener presentes en los procesos de construcción del conocimiento? 
Estas y otras cuestiones han centrado la reflexión y el debate en esta segunda fase de discusión realizada por los autores durante el 2021 que ha dado lugar al presente trabajo. A partir de la explicación de una serie de cuestiones pretendemos analizar las prácticas de participación, interacción y colaboración que favorecen que podamos avanzar a medida que el contexto educativo del siglo XXI se expande y cambia, identificar los principales avances en la creación del conocimiento en contextos digitales, valorar hasta qué punto las conclusiones de 2014 han encontrado un reflejo en lo que ha sucedido desde entonces, y discernir los retos más importantes que pueden aparecer en la investigación e innovación educativa en los próximos 5 años.

\section{Retos de la investigación educativa en la construcción del conocimiento en contextos digitales}

En base a lo expuesto anteriormente, se reflexiona a continuación sobre algunos de los retos que, sin ánimo de ser concluyentes, pueden marcar tendencias en los procesos educativos actuales que se desarrollan en contextos digitales: la democratización de la educación relacionada con la diversidad en sentido amplio; las implicaciones de la pandemia Covid-19 en lo que concierne a los modelos educativos y las necesidades generadas; el aprovechamiento de las potencialidades que ofrecen los múltiples y diversos contextos de creación de conocimiento y la articulación fluida entre ellos; el conocimiento generado y compartido por la curación de contenidos y el papel de los algoritmos automatizados; y las analíticas del aprendizaje y la minería de datos educativos para informar la toma de decisiones basadas en inteligencia artificial. Cada uno de estos retos sugieren cuestiones importantes que deberían ser consideradas por la investigación y la innovación educativa en los próximos años.

\subsection{Democratización de la educación y diversidad en sentido amplio}

Algunos de los retos principales a los que se enfrenta la sociedad en el actual momento social y tecnológico en lo que se refiere a los procesos de creación de conocimiento tienen que ver con cuestiones relacionadas con la diversidad entendida de forma amplia. Son retos relacionados con las condiciones de partida (como, por ejemplo: intereses, aptitudes, estadios de desarrollo, etc.), los roles de trabajo y las subjetividades (cultura, creencias, realidades contextuales, etc.) de las personas que se implican en estos procesos de creación y que, de forma inevitable, se reflejan en el conocimiento que se crea (Peters, Besley y Jandric, 2018). Estos retos apuntan a la educación y a la tecnología de forma directa como contexto en tanto que sus consecuencias aparecen de forma muy obvia en los entornos específicamente dedicados a ellas, pero también apuntan a ambas como herramientas para enfrentarlos y como espacios donde encontrar vías de solución.

La diversidad es una realidad innegable en un mundo donde la producción del conocimiento se ha abierto a contextos más amplios y ha trascendido los límites habituales. Hoy esa diversidad se plasma en múltiples formatos que van más allá de los documentos escritos o visuales y que han conseguido un desarrollo sin precedentes de contenidos multimedia, hipermedia y de realidades mixtas. Además, las narrativas se han expandido y diversificado gracias a conocimiento creado y compartido en hilos de Twitter, stories de Instagram, remixes de Tiktok, así como a las formas y lugares de producción, siendo más habitual que nunca la producción de conocimiento de forma 
colaborativa por personas que se encuentran en muy diversos lugares y realidades del mundo y a los cuales se les atribuye autoridad -y poder-, antaño concentrado en contextos muy precisos.

Esta diversidad plantea, al menos, una triple exigencia de respuestas educativas y de ambiciones tecnológicas. En primer lugar, exige un compromiso real con la accesibilidad de todos los contenidos y con el diseño universal como estándar innegociable de todas las propuestas educativas (Ebersold, 2021), y no solo como concesión extraordinaria de algunos de nuestros recursos. Este compromiso implica que el objetivo de accesibilidad deje de ser un horizonte deseable para convertirse en una exigencia, por ejemplo, de que todo el conocimiento abierto que se genera desde las instituciones sea realmente abierto para toda la ciudadanía. Pero, además, implica que los grupos que no son "usuarios habituales" de las instituciones sepan de la existencia de ese conocimiento y reciban todo el apoyo necesario para acceder a él con facilidad.

Esta apuesta por la diversidad implica también un reconocimiento explícito del papel de grupos de personas tradicionalmente no reconocidos en la producción de conocimiento y de los formatos no considerados suficientemente relevantes por no formar parte de la tradición cultural (Longino, 2017). Es necesario, para ello, que se haga patente y además se favorezca un entorno educativo y tecnológico que priorice el reconocimiento de todas las personas implicadas en la autoría (no solo el primer autor, como en la mayoría de los estándares de citación más utilizados), de la realidad de la persona que enmarca esa autoría (género, procedencia, edad, contexto) y de la importancia de la variedad en formatos y enfoques (Llopart \& Esteban-Guitart, 2018). Entendemos que los actuales modelos de reconocimiento de la autoría son reflejo de un modelo de producción de conocimiento casi directamente importado de la edad moderna -si no de la edad media-, donde la producción de conocimiento se concentraba en núcleos urbanos y académicos concretos, y la difusión de ese conocimiento debía ajustarse a la escasez de espacio en el papel. Hoy la producción es distribuida, como hemos mencionado más arriba, y, aunque sigue siendo prioritario evitar el despilfarro de papel, la gran mayoría de las producciones se hacen y difunden en electrónico, con las posibilidades extra de espacio que eso ofrece.

Desde una perspectiva más epistemológica, uno de los principales retos educativos a los que nos enfrenta este nuevo escenario de producción del conocimiento tiene que ver con la necesidad de emprender procesos reales de descolonización de los discursos y de resignificación de las llamadas epistemologías del Sur (Santos, 2018). Los grupos que históricamente han sido oprimidos en todo el mundo siguen siendo mayoritariamente ignorados y sus luchas silenciadas en el modelo de producción de conocimiento actual. Este modelo se considera heredero de los modelos epistemológicos y coloniales "del norte" que han sido hegemónicos prácticamente hasta la actualidad en casi todas las áreas de producción del conocimiento, priorizando unas visiones particulares del conocimiento y del mundo sobre otras (Nieto, 2018). Esta hegemonía de los modelos del norte ha llegado sin problema alguno al conocimiento generado en esta nueva era tecnológica y, a pesar de que la posibilidad de la pluralidad en esos discursos es mayor, lo cierto es que hemos reproducido los patrones de colonialismo en la era del conocimiento (Lambert \& Czerniewicz, 2020; Morozov \& Pavlova, 2018).

A la luz de esta realidad, resulta imprescindible contribuir desde la educación a la generación de modelos alternativos de producción de conocimiento y al pensamiento 
crítico (Abdi et al., 2015; Yedaide \& Porta, 2020). Se trata de generar nuevos procesos de creación y nuevos modelos de pensamiento que no solo incluyan miradas múltiples, sino que partan de realidades culturales diversas vistas desde esas realidades. Es decir, no se trata de mirar a otras culturas e incluirlas en "nuestro" discurso tamizadas por nuestra visión cultural de su valor, o que les proporcionemos nuestra visión de lo que es valioso para que "mejoren" su posición global asimilando nuestras visiones culturales. De lo que se trata es de establecer diálogos críticos realmente simétricos entre sujetos que analizan realidades globales desde visiones diversas, fruto de la experiencia de las personas que viven subjetividades locales que les proporcionan perspectivas únicas. Ahora bien, tampoco es cuestión de aceptar acríticamente formaciones discursivas presentadas como conocimiento con el mero argumento de que han sido producidas mediante modelos o epistemologías alternativas. La cuestión es atender críticamente y plantearnos qué entendemos por conocimiento y provocar procesos de intercambio y consenso -de pensamiento- en contextos multiculturales y multilocales que generen conocimiento diverso y pensamiento asertivo y crítico.

Esto debería reflejarse en una revisión profunda del significado de los términos enseñar, aprender y evaluar y, cómo no, de las prácticas asociadas a ellos dentro y fuera de las aulas (Martín \& Artiaga, 2017). Esta revisión parece ser una preocupación constante de la didáctica de los últimos tiempos, aunque suele tener enormes problemas para generalizarse en las prácticas curriculares y tampoco parece ser prioridad de las prácticas de la educación con tecnología (Zawacki-Richter et al., 2019). La revisión debe profundizar en modelos didácticos más flexibles y más críticos en todos los niveles educativos (incluida, por supuesto, la formación inicial de los docentes), que partan de las realidades e intereses de los estudiantes y las entiendan en su complejidad tecnológica; que, por ejemplo, propongan tareas de exploración de contextos diversos en tareas de colaboración; o que, en vez de pretender la asimilación acrítica, se esfuercen por cuestionar de forma crítica el conocimiento producido, que evalúen la producción de conocimiento en formatos diversos y con estructuras poco tradicionales; y que entiendan el papel fundamental que las tecnologías tienen en esas propuestas no solo como instrumento, sino también como contexto y como condicionante (Esteve-Mon, Castañeda y Adell, 2018).

Además, este tipo de planteamientos requieren de una apuesta más decidida por experiencias y programas generalizados de intercambio y colaboración que entiendan a la otra persona en su realidad, que abran procesos reales de intercambio en condiciones de igualdad, tanto a nivel escolar como a nivel profesional y académico (abundar en propuestas de proyectos intercentros, nacionales e internacionales de intercambio apoyados por tecnología, programas de movilidad -física o virtual- de estudiantado y profesorado), y que eviten de forma explícita las perspectivas paternalistas, y colonizadoras al fin, que hemos priorizado hasta el momento (Asher, 2010; Santos \& Martins, 2021).

\subsection{Implicaciones de la pandemia COVID-19 en la educación}

La pandemia ha transformado la enseñanza de nuestras instituciones educativas desde modelos fuertemente anclados en la concepción transmisiva de la formación y en la presencialidad docente-discente a una situación fuertemente mediada por las tecnologías digitales $\mathrm{y}$, fundamentalmente, por Internet. Dicha transformación ha supuesto la 
aparición de una serie de retos: las limitadas competencias digitales de ciertos docentes, la falta de infraestructuras, la falta de recursos digitales, trascender el modo verbal y escrito e incorporar lenguajes multimodales y digitales, como ya se ha comentado en el apartado anterior, o la ausencia de orientaciones para la atención remota de los estudiantes (Inciarte et al., 2020).

Las creencias y actitudes que el docente tenga respecto a las posibilidades que las tecnologías le pueden ofrecer para su desarrollo profesional son clave, no solo para que las incorpore a su práctica, sino también para la forma en la cual lo hace. Y la situación docente que se creó, bien a distancia o posteriormente en lo que se denominó como enseñanza híbrida, puso de manifiesto que muchos docentes no se encontraban cómodos con la utilización de las tecnologías, aumentándose la angustia, el estrés y la ansiedad (Casimiro et al., 2020), y que en muchos casos su utilización se concentró en dos aspectos: considerar la plataforma puesta a su disposición como repositorios de objetos de aprendizaje y convertir las sesiones de clase en sesiones de vídeoclase, en lugar de utilizar las tecnologías como herramientas de innovación y colaboración.

Otro reto emergente durante la pandemia ha sido la estrategia de seguimiento y evaluación a emplear con los estudiantes. La dificultad para adaptarse a los contextos a distancia e híbridos ha puesto de manifiesto que muchos docentes siguen apoyándose en un único modelo de evaluación de sus estudiantes con pruebas escritas específicas y realizadas puntualmente, evidenciando que el modelo de evaluación continua por parte de los docentes no es ampliamente empleado ni asumido.

A pesar de lo indicado, se han identificado una serie de prácticas innovadoras en la aplicación de las tecnologías digitales por parte de los docentes durante la pandemia que sería interesante evaluar e investigar. Dichas prácticas favorecen el aprendizaje autónomo y autorregulado por los estudiantes, así como las acciones formativas colaborativas y la transformación de las prácticas escolares (Ellis et al., 2020; Kidd y Murray, 2020; Bas et al., 2021; Morocho, 2021; Trujillo, 2021).

Estas buenas prácticas también han señalado indirectamente un aspecto que se viene poniendo de manifiesto en las investigaciones en TIC: que éstas por sí solas no mejoran el aprendizaje, sino que la mejora depende de una serie de variables como el currículum, el profesor, o hasta las estrategias que se apliquen. No se debe caer en el error, por tanto, de pensar que, si bien la pandemia ha acelerado la adopción de tecnologías, estas se han integrado en el currículum (Ewing y Cooper, 2021).

La falta de recursos tecnológicos existentes en los hogares, la falta de recursos flexibles y adaptados a los programas y contenidos y la calidad de la conexión a Internet han condicionado la transición a la educación a distancia y la aplicación de modelos híbridos. Esto ha repercutido en la disminución de la calidad de la experiencia de aprendizaje realizada y en el aumento de la deserción escolar por parte del alumnado (Cano et al., 2020; Burgos, Vázquez-Cano, López-Meneses y Adaos, 2021), sobre todo en el caso de los alumnos pertenecientes a colectivos más vulnerables social y económicamente (Schleicher, 2021; Aditya, 2021; Álvarez-Álvarez. y García-Prieto, 2021), como se ha señalado en el apartado dedicado a la democratización de la educación y a su relación con la diversidad entendida en un sentido amplio. 
Los estudiantes también encontraron dificultades reflejándose en un similar desconcierto e inseguridad al trabajar en los contextos tecnológicos, fundamentalmente debido a las condiciones de conectividad y al mito de que los alumnos, por el hecho de haber nacido en un período histórico determinado, son por definición nativos digitales y por tanto competentes digitales.

Los aspectos mencionados plantean una serie de retos a las investigaciones futuras en el terreno de las tecnologías digitales. Uno de ellos es indagar en las competencias digitales de los docentes, poniendo en acción programas de desarrollo de competencias TIC y evaluando su eficacia, de forma similar a lo que, como veremos más adelante, convendría hacer respecto a las competencias relacionadas con el uso de las analíticas de aprendizaje. Algunos trabajos han señalado que, como resultado de la experiencia en la pandemia, los docentes mejoraron su capacitación tecnológica y han cambiado las imágenes que tenían sobre las TIC (Beardsley et al., 2021; Navarro-Espinosa et al., 2021; Scully, Lehane y Scully, 2021). En este sentido, podría ser de utilidad realizar estudios comparativos respecto a sus competencias precovid y postcovid.

Diferentes investigaciones ponen de relieve que la falta de capacitación digital docente ha sido compensada en algunos casos mediante la creación de un clima afectivo basado en la empatía, el optimismo, la retroalimentación y la motivación constante a los estudiantes (Maile, Mena y Feinauer, 2020; Ryan, 2021; Yates et al., 2021). Ello llevaría a establecer una línea futura de investigación referida a las competencias que los docentes deben tener para crear un clima psicoafectivo en los entornos de formación a distancia.

Diferentes líneas de investigación pueden abrirse para analizar el "parque" tecnológico al que han podido acceder los estudiantes, los problemas con que se han enfrentado y los resultados obtenidos. Así, sería interesante investigar en profundidad la brecha digital tecnológica a la cual se han enfrentado los estudiantes para poder establecer de este modo políticas compensatorias y adoptar medidas que garanticen un acceso adecuado a las tecnologías (e.g., mejorar la conexión, seleccionar plataformas virtuales amigables y flexibles, proporcionar recursos tecnológicos a los hogares que carecen de ellos.

También en relación con los estudiantes, se hace necesario realizar investigaciones que indiquen el grado real de las competencias digitales que poseen y proponer acciones formativas en consecuencia. Especialmente teniendo en cuenta otras alfabetizaciones a las que han podido llegar por procesos no formales e informales de creación del conocimiento como los mencionados en el apartado anterior de este informe. Ello se hace necesario, sobre todo, si tenemos en cuenta que los alumnos más vulnerables han podido resultar especialmente perjudicados durante la pandemia. Las experiencias que han tenido con las TIC, algunas de las cuales no han sido muy exitosas, plantean el reto de investigar su predisposición a aprender con las TIC tras estas experiencias.

También se abre el reto de investigar las repercusiones que la formación a distancia ha tenido en los aprendizajes alcanzados por los estudiantes y su deserción escolar. Diferentes investigaciones realizadas durante la COVID-19 han informado de la posible pérdida de aprendizaje de los estudiantes como consecuencia de la formación a distancia. Engzell, Freya y Verhagen (2021) han documentado una pérdida de tres puntos porcentuales, lo que equivaldría a una quinta parte del curso escolar, siendo la situación más preocupante la de estudiantes de bajos recursos económicos. Por su parte, Kuhfeld y 
otros (2020), realizando proyecciones sobre las pérdidas de aprendizaje basados en estudios anteriores sobre absentismo y análisis del aprendizaje en verano, realizan una proyección según la cual se alcanzaría una pérdida en lectura entre el 63 y el 68\%, y en matemática entre el 37 y el $50 \%$.

Lo comentado anteriormente puede servir para establecer medidas correctoras, algunas de las cuales pueden consistir en estudiar las posibilidades de las TIC para la construcción de materiales para el aprendizaje autónomo por parte de los estudiantes.

Como ya se ha apuntado, durante la pandemia también se han llevado a cabo experiencias innovadoras en el uso de las TIC. Valdría la pena investigar estas experiencias, algunas de las cuales pueden considerarse "buenas prácticas", para analizar las posibilidades de transferencia a contextos presenciales e híbridos, y los cambios que podrían producirse en el modelo pedagógico de los docentes y en la aplicación de nuevas estrategias y prácticas docentes como la gamificación, la clase invertida, el escape room o la realidad mixta. En este análisis debería tenerse en cuenta si la ubicación geográfica del centro o su titularidad ha establecido diferencias en la aplicación de las TIC.

Relacionado con lo anterior, podría ser interesante analizar los cambios que las TIC introdujeron en los momentos de pandemia y las lecciones que de ellos se pueden derivar para acelerar los procesos de cambio en la enseñanza y el aprendizaje (Ellis, Steadman y Mao, 2020). También puede ser una fuente importante de información el estudio de la utilización específica de las tecnologías digitales que han hecho los docentes para adaptar las actividades de aprendizaje al contexto familiar, a los recursos domésticos de los que disponían los estudiantes y al papel que la familia podía jugar en esta situación de aprendizaje, como se señala en el siguiente apartado del informe.

Con el objeto de facilitar la incorporación de las tecnologías digitales como elemento de equidad social, sería de especial utilidad investigar el grado de utilización de tecnologías gratuitas y de código abierto en los momentos de la pandemia, así como analizar los motivos de su utilización, o no, por los docentes. Así mismo, se hace necesario profundizar en lo ocurrido en la pandemia con los estudiantes de educación inclusiva, pues pocos son los trabajos que al respecto se han realizado (Aloizou et al., 2021; Parmigiani et.al., 2021).

Cabe señalar también la posibilidad de iniciar una línea de investigación para analizar las respuestas dadas por los centros educativos en la situación de pandemia, e indagar en las decisiones organizativas adoptadas, los modelos de interacción docente-estudiante y docente-familia que han surgido, las tecnologías utilizadas y la forma en que se movilizaron, entre otros aspectos. Finalmente, y para cerrar este apartado, convendría estudiar los cambios que ha supuesto este proceso "intensivo" de incorporación de las tecnologías digitales para el desarrollo de las instituciones educativas y las transformaciones que han tenido que hacer para ello y las decisiones administrativas y de autonomía adoptadas.

\subsection{Articulación fluida de los contextos de construcción y creación de conocimiento}

Una de las características más sobresalientes de la nueva ecología del aprendizaje (Barron, 2006; Coll, 2013; Ito et al., 2013) es la existencia de múltiples y diversos 
contextos de actividad que ofrecen oportunidades y recursos para aprender a quienes participan en ellos. Por supuesto, no todos los contextos ofrecen las mismas oportunidades y recursos para aprender ni las oportunidades y recursos que ofrecen a quienes acceden a ellos tienen el mismo valor ni el mismo impacto sobre los procesos de desarrollo, socialización y formación de las personas. En este escenario, ya no es posible seguir entendiendo el aprendizaje como un proceso que tiene lugar fundamentalmente, si no exclusivamente, en el contexto de la educación formal. El aprendizaje, y con él la construcción y la creación del conocimiento, pueden tener lugar en múltiples contextos de actividad, educativos o no, y su valor personal y social ya no está indisolublemente asociado a un contexto espacio-temporal preciso como es, por ejemplo, el aula de una institución educativa.

Esta conciencia de la diversidad de los espacios para aprender, así como del desbordamiento de los contextos tradicionales de aprendizaje, parte también de la noción contemporánea del aprendizaje como una "actividad emergente" (de Laat y Dohn, 2019). Es decir, entendemos que las personas no aprenden porque se les administre un determinado "tratamiento" en forma de "diseño instruccional", sino que, si bien el diseño sitúa la actividad de las personas, la actividad de aprendizaje emerge o no como resultado de las interacciones de las personas con el contexto (las tareas que emprende, las relaciones sociales que la sustentan y las condiciones materiales que la soportan) (NLEC, 2021; Goodyear et. al. 2021).

En el primer apartado hemos hablado de la diversidad como elemento fundamental de la generación de conocimiento en nuestros días, y esa misma diversidad se refleja casi de forma íntegra en nuevos o emergentes contextos situados de aprendizaje. Contextos que están en función de la situación personal de los aprendices y dependen de factores como desde qué lugar o entorno material aprenden, cuál es su historia como aprendices, en qué momento personal, social, y familiar aprenden, etc., que se sitúan además en la inmensa variedad de tareas que pueden emprenderse hoy y que, gracias al momento actual de desarrollo tecnológico y penetración de las tecnologías en la sociedad (ancho de banda, conexiones inalámbricas, acceso móvil, capacidad de procesamiento de los dispositivos), superan las posibilidades que teníamos hace sólo una década haciendo que el trabajo multimodal en equipo y la colaboración en línea en tiempo real sean una realidad para una buena parte de la sociedad que se conecta.

El hecho de que el aprendizaje pueda tener lugar en múltiples contextos, sin embargo, no garantiza que lo tenga; y sobre todo no garantiza que todas las personas tengan acceso a los contextos de actividad que ofrecen mayores y mejores oportunidades y recursos para aprender. La multiplicidad y diversidad de contextos de actividad presentes en nuestra sociedad que ofrecen oportunidades y recursos para aprender abren enormes posibilidades desde el punto de vista del aprendizaje. Pero abren también la puerta, en contrapartida, a nuevas y potentes fuentes de desigualdad, en la medida en que el acceso a los contextos de actividad que ofrecen una mayor riqueza de oportunidades y recursos para aprender está fuertemente asociado a factores de naturaleza socioeconómica. Con el agravante de que son desigualdades ante el aprendizaje que tienen su origen y responden a situaciones que se sitúan más allá de las instituciones de educación formal y cuyo abordaje y tratamiento requieren, en consecuencia, el diseño y la puesta en marcha de actuaciones que, si bien deben seguir teniendo estas instituciones como epicentro, no pueden limitarse a ellas. 
Las consideraciones precedentes permiten entender la importancia concedida en el pensamiento pedagógico actual y en la investigación educativa y psicoeducativa recientes a esta multiplicidad y diversidad de contextos de actividad en los que podemos aprender, a su conformación, características y componentes, a su articulación y, muy especialmente, a cómo aprovechar su potencialidad para promover la realización de aprendizajes más sólidos, significativos y profundos y, al mismo tiempo, evitar los riesgos que también comportan. Situados en estas coordenadas, comentaremos brevemente a continuación tres vertientes de este ámbito de investigación y de reflexión relativas, respectivamente, a (i) las dimensiones que pueden intervenir en la conformación de estos contextos de actividad y que hay que tener en cuenta consecuentemente en su análisis y diseño; (ii) la articulación de los contextos de actividad escolares y no escolares en el marco de una visión distribuida e interconectada del aprendizaje; y (iii) la utilización de las tecnologías digitales de la información y la comunicación como herramientas de acompañamiento y ayuda a los aprendices en los procesos de construcción, revisión y re-construcción de sus trayectorias personales de aprendizaje.

En lo que concierne a la conformación, dimensiones y características de los contextos de actividad, el hecho más destacable desde el punto de vista de los procesos de aprendizaje es, a nuestro juicio, la evolución desde el interés por los construidos y organizados en torno a una dimensión -contextos escolares versus no escolares, presenciales versus a distancia, físicos versus en línea, analógicos versus digitales, síncronos versus asíncronos, basados en un solo medio versus multimedia, etc.- hacia el interés por los conformados en torno a la interrelación de varias dimensiones -por ejemplo, los contextos, escolares o no escolares, en los que se desarrollan actividades de aprendizaje que combinan momentos de interacción presencial cara a cara con el trabajo grupal en línea síncrono y asíncrono-. La razón última de esta evolución se encuentra en el abanico de posibilidades y alternativas que estos contextos ofrecen para el diseño y desarrollo de los procesos de aprendizaje, ya que permiten aprovechar las ventajas y compensar las limitaciones que presentan los contextos organizados en torno a cada una de las dimensiones por separado. Esta multidimensionalidad permite, entre otras cosas, combinar diferentes lenguajes y formatos de representación de la información y del conocimiento, así como adoptar diferentes formas de organización de la actividad conjunta de los participantes, lo cual, como ha puesto de manifiesto la investigación desarrollada en el marco de las ciencias del aprendizaje (Darling-Hammond et al., 2020; Sawyer, 2015), son factores propicios para la realización de aprendizajes con un alto grado de significatividad.

La investigación sobre el aprendizaje ha puesto también de manifiesto que el establecimiento de conexiones entre aprendizajes y experiencias de aprendizaje que han tenido lugar en diferentes lugares y momentos es un factor clave para que los aprendices profundicen en sus aprendizajes y otorguen sentido y valor personal a lo aprendido (Barron, 2010; Coll, Esteban-Guitart e Iglesias, 2020; Ito et al., 2020), lo cual nos conduce directamente a la segunda vertiente del tema antes mencionada: la necesidad de que exista una articulación de los contextos de actividad escolares y no escolares en el marco de una visión distribuida e interconectada del aprendizaje. Las conexiones, sin embargo, no se establecen siempre ni necesariamente de forma natural y espontánea. El aprendizaje está cada vez más distribuido entre diferentes contextos de actividad, pero no siempre lo que se aprende en un contexto se relaciona y se conecta con lo que se aprende en los otros, dando lugar así a menudo a compartimentos estancos que dificultan la funcionalidad y utilización de los aprendizajes realizados. Cabe, pues, afirmar que, si bien actualmente el 
aprendizaje está ampliamente distribuido, en cambio suele estar escasamente interconectado. Este desajuste es especialmente preocupante en la medida en que, si bien los aprendizajes y las experiencias de aprendizaje son la materia prima para la construcción de las trayectorias individuales de aprendizaje, las conexiones que establecemos entre ellas son la argamasa que les da consistencia y continuidad y las convierte en un elemento vertebrador de la identidad personal (Esteban-Guitart, 2021). De ahí la importancia de poner el foco de la acción educativa en el seguimiento, acompañamiento y ayuda a los procesos de construcción, revisión y reconstrucción de trayectorias personales de aprendizaje ricas, potentes y habilitadoras de nuevos aprendizajes, lo cual nos lleva directamente a la tercera de las vertientes a la que aludíamos al inicio de este apartado.

El tránsito desde un modelo educativo centrado en la escolarización y en el aprendizaje escolar hacia un modelo que pone el foco de la acción educativa en ayudar a los aprendices a construir trayectorias personales de aprendizaje que les habiliten para seguir aprendiendo en el futuro encuentra en las TDIC un aliado esencial para avanzar en la dirección deseada (Esteban-Guitart, Coll \& Penuel, 2018). Por una parte, los contextos de actividad que ofrecen nuevas e inéditas oportunidades y recursos para aprender se han conformado a menudo a partir de, o en torno a, estas tecnologías -baste recordar, a título de ejemplos, las comunidades virtuales de interés, de práctica o de aprendizaje, los repositorios de prácticas educativas abiertas o las aplicaciones de realidad virtual-, lo que las convierte en elementos fundamentales para comprender los procesos de aprendizaje que en ellos tienen o pueden tener lugar. Por otra parte, estas tecnologías son a menudo imprescindibles para poder realizar el seguimiento y acompañamiento de los aprendices en su transitar por diferentes contextos de actividad, registrar y documentar las experiencias de aprendizaje fruto de este tránsito, y establecer conexiones entre ellos superando las barreras físicas, temporales e institucionales que a menudo los separan. Sin duda, las TDIC han jugado un papel determinante en la aparición de nuevos contextos de actividad que ofrecen recursos y oportunidades para aprender-así como en el incremento de la potencialidad como espacios de aprendizaje de contextos de actividad tradicionales como, por ejemplo, el familiar, el laboral, el cultural o el escolar-que es uno de los rasgos más característicos de la nueva ecología del aprendizaje. Todo induce a pensar que en un futuro inmediato van a jugar un papel igualmente destacado en la concreción del nuevo modelo de educación distribuida e interconectada que se necesita en este escenario.

\subsection{Curación de contenidos}

La curación de contenidos se conceptualiza como la práctica de crear, identificar y compartir recursos multimodales con implicaciones para la identidad y la autorrepresentación (Potter, 2011). A medida que surgen las pedagogías abiertas, las prácticas de curación de contenido pasan a un primer plano de la creación de conocimiento y la agencia del aprendiz (Baran \& AlZoubi, 2020). En una visión del aprendizaje que trasciende la recepción pasiva de información, la interpretación y reedición de la información son etapas imprescindibles, sea cual sea el abordaje pedagógico. La curación en entornos digitales incluye la escritura y la creación, pero se extiende para incluir la recopilación, catalogación, ensamblaje y exhibición de marcadores de identidad (Potter, 2011). Pero recopilar es más que buscar en Internet una gran variedad de recursos. Ahora, más que nunca, la curación exige un consumidor crítico

Construir el conocimiento en la era digital: retos y reflexiones. M. Marimon-Martí, J. Cabero, L. Castañeda, C. Coll, J. M. de Oliveira, M. J. Rodríguez-Triana. 
y exigente, y los consumidores críticos y exigentes exigen también una curación de similares características (Sawyer, 2014).

La curación de contenido, antaño labor manual y determinada casi en su totalidad por la propia experticia de los sujetos, ahora es asumida de forma prácticamente imperceptible por algoritmos automatizados que operan de forma casi inevitable, desde la práctica totalidad de las tecnologías. Hace poco más de diez años descubríamos las maravillas de almacenar, clasificar, organizar y recuperar información relevante en la nube y poder acceder a ella desde cualquier lugar, y con esa posibilidad nuestra capacidad de reflexión sobre esa información se convertía en una actividad permanente que además enriquecía nuestros archivos y la percepción misma del aprendizaje. Además, las herramientas de la Web 2.0, especialmente dotadas para la curación, permitieron que este proceso de curación fuese colectivo y compartido, y el éxito inicial y determinante de herramientas como Twitter, o como el ahora desaparecido Friendfeed (https://en.wikipedia.org/wiki/FriendFeed), se basaban en la posibilidad de acceder a la curación de otros y entenderla como conocimiento creado en el nuevo entorno, que era además de utilidad para nosotros.

Sin embargo, la web 2.0 ha sufrido una tremenda transformación. Lo que antes era el ágora por excelencia de nuestra aldea global, llena de espacios para interactuar y para curar contenido, se parece cada vez menos a esa imagen de diversidad y ha perdido, al menos en parte, su característica más preciada en lo que se refiere a curación entre iguales: ha dejado de ser tan distribuida como entonces (https://ia.net/topics/web-trend-map2018). En su lugar, la Internet de hoy es un espacio monopolizado por empresas que ofrecen servicios, o colecciones de servicios conectados, que -de manera a veces imperceptible para el propio usuario- eligen previamente lo que el usuario "consumirá" y le hacen una "preselección" de contenidos, supuestamente en base a sus preferencias. En este nuevo entorno, la curación humana de contenido es un reto, porque debe superar los condicionantes de la pre-curación de contenido hecha por la máquina, de manera que la generación y conservación de conocimiento en forma de colecciones de contenido curado es cada vez más una utopía de momentos tecnológicos anteriores.

Posicionar a los estudiantes como curadores implica reconocimiento de los múltiples procesos involucrados al crear significados multimodales utilizando medios digitales. Estos incluyen la deliberación y la criticidad en la implementación del conocimiento de los dispositivos; la selección, combinación y disposición de una variedad de recursos de creación de significado (Cloonan et al., 2019). La curación de significados multimodales que los medios digitales hacen posible permite la experiencia reflexiva a través de diferentes lenguajes, no únicamente el lenguaje verbal escrito. El proceso de selección tiene el potencial de apoyar el aprendizaje auténtico y contribuir a la mejora del conocimiento y las habilidades propias del siglo XXI de los estudiantes (Baruch \& Gadot, 2021). Requiere habilidades personales y sociales, como habilidades cognitivas y metacognitivas desde una perspectiva individual y social, habilidades sociales $\mathrm{y}$ habilidades afectivas (Baruch \& Gadot, 2021). Entre los desafíos a los que nos enfrentamos para posicionar a los estudiantes como curadores podemos citar, por ejemplo, el desarrollo de procesos metacognitivos complejos, que a menudo requieren tiempo y reflexión no habitualmente dispensados en tiempos de instantaneidad. 
Especial atención merece la formación del profesorado. Los maestros del siglo XXI participan con frecuencia en la conservación de recursos educativos en línea (Liu et al., 2020; Torphy et al, 2020). Es importante educarlos en estrategias de curación para que los recursos seleccionados por maestros tengan una alta demanda cognitiva ( $\mathrm{Hu}$ et al, 2020) y favorezcan un compromiso profundo con una educación culturalmente relevante (Hu et al., 2019). Sawyer et al. (2019) proporcionan evidencia de que los futuros docentes deben capacitarse en el desarrollo intencional de las habilidades de curación, reconociendo y modelando la profundidad y amplitud de los recursos, incluidos aquellos que no están necesariamente sancionados. Aunque no se espera que los profesores y los futuros profesores reinventen la rueda, se espera que den crédito a las fuentes consultadas y piensen críticamente sobre las fuentes que utilizan (Sawyer, 2019). Entre las estrategias relevantes para capacitar a los futuros docentes en la curación de contenidos, podríamos mencionar: i) el conocimiento y desarrollo de criterios de evaluación de contenidos; ii) el análisis crítico y debate de la calidad de recursos educativos encontrados en línea; y iii) la creación de entornos personales de aprendizaje como resultado de procesos reflexivos guiados. A modo de ejemplo, el primer punto del área "Facilitar la competencia digital de sus estudiantes" del modelo 1DigCompEdu (Cabero \& Palacios, 2020) se refiere a la "Información y alfabetización mediática".

Como se ha comentado, en un entorno de comunicación caracterizado por la multimodalidad generalizada y la cultura digital, las prácticas de creación e intercambio de contenido ya no pueden describirse completamente por los modos de significado abarcados por el discurso escrito comunicación escrita y el discurso oral (Kress, 2003). De hecho, Kress y Selander (2012) argumentaron que limitar las respuestas de texto de los estudiantes al modo lingüístico subestima las posibilidades que ofrecen los medios digitales, lo que a su vez puede tener un impacto en la confianza de los estudiantes en el conocimiento de la institución educativa. Por el contrario, cuando la curación penetra las prácticas educativas, los alumnos pueden posicionarse como participantes activos de la investigación con oportunidades para influir en las pedagogías del aula (Cloonan, 2020). En prácticas educativas permeables a la curación de contenidos, los alumnos dejan de ser consumidores de los recursos ofrecidos por los docentes y se convierten en actores que aportan elementos para análisis de todo el grupo. Sus intereses cobran relevancia en la selección de contenidos y pasan a tener la potencialidad de influenciar el aprendizaje propio y colectivo. El currículum, en estas prácticas, deja de ser determinado de arriba hacia abajo y pasa a contemplar las experiencias, expectativas y visiones del mundo de los propios estudiantes.

\subsection{Analíticas de aprendizaje para informar la toma de decisiones en educación}

Los campos de investigación de las analíticas del aprendizaje y la minería de datos educativos han adquirido gran inercia en los últimos años, durante los cuales se han propuesto múltiples soluciones para comprender y optimizar el aprendizaje y los contextos donde éste tiene lugar ${ }^{1}$. Dichas soluciones abarcan desde la mera visualización de indicadores hasta los agentes para la toma automática de decisiones basados en

1Definición propuesta por SOLAR: https://www.solaresearch.org/about/what-is-learning-analytics 
inteligencia artificial (AI, del inglés Artificial Intelligence) (Schwendimann et al., 2017; Vieira, Parsons \& Byrd, 2018; Du et al., 2021).

En relación a los modelos emergentes de construcción del conocimiento en contextos digitales, estos campos de investigación (i.e., LA, EDM y AI) han contribuido a una mejor comprensión de los procesos de participación, interacción y colaboración. Sin embargo, existen aún múltiples retos que han de ser afrontados en el corto, medio y largo plazo.

A nivel tecnológico, surgen múltiples desafíos. En primer lugar, y en relación a la existencia de múltiples y diversos contextos mencionada en el punto 3, la naturaleza multimodal del aprendizaje y su ubicuidad (véase, por ejemplo, el caso del aprendizaje en contextos laborales) requiere que, al analizar los procesos de participación, interacción y colaboración, estos aspectos sean tenidos en cuenta. Esto implica que hemos de ir más allá de la evidencia basada exclusivamente en logs, la cual ha sido la tendencia mayoritaria, y debamos integrar múltiples fuentes de datos que nos ayuden a proporcionar una visión más completa del proceso de aprendizaje (Papamitsiou \& Economides, 2014; Schwendimann et al., 2017; Viberg et al., 2018; Samuelsen, Chen \& Wasson, 2019; Crescenzi-Lanna, 2020; Ifenthaler \& Yau, 2020; Du et al., 2021). Aunque son múltiples las complicaciones que surgen (e.g., en relación a la recogida, integración, análisis y visualización de datos multimodales), muchos son ya los investigadores trabajando en esta problemática (conocida en inglés como Multimodal Learning Analytics) (Vieira, Parsons \& Byrd, 2018; Samuelsen, Chen \& Wasson, 2019; Crescenzi-Lanna, 2020; Du et al., 2021). Entre las propuestas existentes podemos encontrar sistemas que proporcionan retroalimentación al alumno sobre sus habilidades de presentación en público que toman como fuente la voz, la posición y el movimiento (Ochoa et al., 2020); herramientas para la regulación de la colaboración basadas en la conversación y el texto co-creado por los alumnos (Chejara et al., 2020); herramientas para evaluar el rendimiento cognitivo utilizando aspectos fisiológicos y faciales (Sharma et al., 2020); y aplicaciones que utilizan sensores de movimiento y posición para dar soporte al aprendizaje en el trabajo, como puede ser el caso de la medicina y la enfermería (Di Mitri et al., 2019; Echeverría et al., 2018). Sin embargo, las soluciones disponibles no son aún asequibles (Crescenzi-Lanna, 2020), no han sido suficientemente evaluadas (Ifenthaler \& Yau, 2020) o no son operativas para su uso en la práctica diaria (Vieira, Parsons \& Byrd, 2018; Romero \& Ventura, 2020; Pargman \& McGrath, 2021). Otra problemática recientemente planteada es la necesidad de datos longitudinales, de especial utilidad para contextos no reglados como es el aprendizaje a lo largo de la vida (Romero \& Ventura, 2020). Tales estudios longitudinales permiten ver el progreso de un individuo a lo largo del tiempo, incluso cuando no hay un gran volumen de datos. Teniendo en cuenta el ritmo actual de progreso, podemos estimar que gran parte de las dificultades tecnológicas serán abordadas en el medio plazo.

A nivel educativo institucional, un problema común es la limitada adopción de las analíticas a pesar de su potencial, especialmente fuera del contexto universitario (Banihashem et al., 2018; El Alfy et al., 2019; Joksimović, Kovanovićv \& Dawson, 2019; Ifenthaler \& Yau, 2020; Valle et al., 2020). En este sentido, cabe mencionar dos requisitos que frecuentemente no se satisfacen: la interpretación (Papamitsiou \& Economides, 2014; Vieira, Parsons \& Byrd, 2018; Crescenzi-Lanna, 2020) y la accionabilidad de las analíticas, i.e., el saber qué hacer con las mismas (Avella et al., 2016; Ifenthaler \& Yau, 2020). En algunos casos, esto puede ser debido a la falta de adecuación con las 
competencias de los usuarios a los que las soluciones van dirigidas (e.g., en relación a la alfabetización en datos de estudiantado, profesorado y otros participantes del proceso educativo) (Papamitsiou \& Economides, 2014; Vieira, Parsons \& Byrd, 2018) o a la falta de transparencia por parte de los proveedores de las soluciones a la hora de explicar a partir de qué datos o cómo se han obtenido los resultados (Avella et al., 2016; Samuelsen, Chen \& Wasson, 2019; Pargman \& McGrath, 2021). Otra causa habitual es la falta de alineamiento entre las soluciones propuestas y las necesidades de los estudiantes, docentes y demás personal educativo que las utiliza (Wong \& Li, 2020), lo cual enlaza con el reto de la diversidad y la necesidad de ofrecer múltiples soluciones a un mismo problema, en lugar de una solución única que satisfaga a todos. Por ello, es crucial tener en cuenta cuáles son las decisiones que tales personas deben tomar e involucrarlas en el proceso de diseño, para así proporcionar información relevante (Rodríguez et al., 2018; El Alfy et al., 2019; Samuelsen, Chen \& Wasson, 2019).

En términos didácticos, otra brecha frecuente es la falta de contexto a la hora de analizar los datos, ya que un mismo resultado puede ser bueno o malo en función del diseño y los objetivos de aprendizaje (definidos por aprendices, en el caso del aprendizaje autorregulado, y/o por agentes educativos, en el caso del aprendizaje dirigido) o el punto del que parta el alumnado (Mangaroska \& Giannakos, 2018; Vieira, Parsons \& Byrd, 2018). Por ello, es importante que las soluciones propuestas tengan en cuenta estos aspectos contextuales para ofrecer un marco de referencia contra el que comparar (Avella et al., 2016; Mangaroska \& Giannakos, 2018). Además, es importante enfatizar el papel que puede jugar la teoría educativa existente en el diseño de las soluciones (Banihashem et al., 2018; Vieira, Parsons \& Byrd, 2018; Viberg et al., 2018; Joksimović, Kovanovićv \& Dawson, 2019; Ifenthaler \& Yau, 2020; Valle et al., 2020). Promover el diseño colaborativo con expertos de diferentes disciplinas contribuiría a una mejor fundamentación teórica de las soluciones propuestas (El Alfy et al., 2019; CrescenziLanna, 2020; Du et al., 2021). Del mismo modo, el codiseño de analíticas con usuarios finales ayudaría a una mejor integración en las prácticas de enseñanza y aprendizaje (Rodríguez-Triana et al., 2018), de forma que estas no se restrinjan exclusivamente a la evaluación del desempeño del estudiantado, sino que se integren también en otros procesos de toma de decisiones, e.g., informando el rediseño didáctico y, de forma fundamental, apoyando procesos metacognitivos y autodirigidos (Roll \& Winne, 2015), de interés incluso para el aprendizaje en contextos laborales (Ruiz-Calleja et al., 2021). Para ello, al igual que en el uso de las TIC mencionado en el segundo punto de este informe, es clave formar a los diferentes usuarios de las analíticas (en especial alumnado, profesorado, familias e instituciones) para que estos sepan cómo integrarlas en su práctica. Estas reflexiones son también aplicables a nivel institucional, en el que el uso de las analíticas de nuevo presenta múltiples oportunidades (e.g., la mejora del curriculum, o la definición de estrategias para detectar y atajar el abandono) pero en el que los objetivos y el punto de partida son esenciales para poder interpretar los resultados (De Silva et al., 2021). Tanto la contextualización (e.g., en el diseño de enseñanza) como la fundamentación teórica, son elementos clave para que las personas sepan qué hacer con los resultados obtenidos, informando así su toma de decisiones, y a la vez pueden ser detonantes que promuevan que esas mismas personas sugieran la obtención de analíticas específicas que sean de su interés. Nuestra expectativa es que satisfacer estos requisitos (i.e., interpretación y accionabilidad) es una cuestión de diseño que puede verse solventada en el corto plazo. 
Desde la perspectiva investigadora, otro problema frecuente es que, aunque son muy numerosos los estudios enfocados en la usabilidad y la aceptación de las soluciones propuestas, aún hay limitada evidencia sobre su impacto en el aprendizaje (Schwendimann et al., 2017). Por ello, es importante prestar especial atención a esta brecha a la hora de evaluar las soluciones propuestas y así construir una base de evidencia sólida para la viabilidad, efectividad y generalización de las soluciones propuestas de forma que se puedan aplicar a otras situaciones y/o contextos (Schwendimann et al., 2017; Viberg et al., 2018; Larrabee et al., 2019; Joksimović, Kovanovićv \& Dawson, 2019; Ifenthaler \& Yau, 2020; Wong \& Li, 2020; Valle et al., 2021). También, y en esta misma línea de investigación, es imprescindible que las investigaciones dedicadas al impacto del uso de analíticas aborden perspectivas que no eludan las condiciones sociales y culturales de las implementaciones, de manera que las propuestas sean cualitativamente más interesantes y provechosas desde el punto de vista educativo.

Finalmente, múltiples son los dilemas éticos que surgen con el uso de las analíticas (Banihashem et al., 2018; Hakimi et al., 2021; Pargman \& McGrath, 2021). Empezando por la recogida de datos, el afán por recoger datos multimodales de los diversos espacios donde el aprendizaje tiene lugar para que el análisis sea más fiable puede derivar en soluciones invasivas e ir en detrimento del aprendizaje y de la privacidad de los individuos (e.g., cuando se utiliza la ubicación de una persona o se recogen medidas biométricas) (Papamitsiou \& Economides, 2014; Avella et al., 2016; Crescenzi-Lanna, 2020; Hakimi et al., 2021). Además, aunque la situación ideal sea recoger datos homogéneos que permitan su posterior análisis, es necesario proporcionar a los individuos la opción de elegir cuáles de sus datos exponer y para qué fines. Otra tensión surge del hecho de exponer datos a terceros. Esto puede entrar en conflicto no sólo con las leyes de protección de datos ${ }^{2}$, sino también con las regulaciones y políticas establecidas a nivel institucional. Para que se cumplan dichas leyes, regulaciones y políticas, uno de los grandes problemas a día de hoy es la necesidad de que los diferentes agentes implicados (e.g., alumnado, profesorado, familias e instituciones) tengan conocimiento de las mismas, las comprendan y sean capaces de discernir qué herramientas no cumplen los requisitos impuestos. Por otro lado, del mismo modo que en el ámbito sanitario los profesionales tienen el deber de intervenir cuando son conocedores de cierta información médica de un paciente, tal responsabilidad aún no está presente en el ámbito educativo. Por ello, es necesario reflexionar sobre a quién estamos exponiendo las analíticas y cuál es la responsabilidad que tales personas tienen al respecto (Hakimi et al., 2021). Pero, para que los usuarios puedan hacerse cargo de sus responsabilidades, de forma similar a lo que sucede con la curación de contenidos, es necesario que estos sean capaces de evaluar hasta qué punto la información que se les presenta es fiable (i.e., en qué datos se basa, su calidad y cómo se ha obtenido) (Papamitsiou \& Economides, 2014; Banihashem et al., 2018; Ifenthaler \& Yau, 2020; Hakimi et al., 2021), de contextualizar los resultados y de contrastar con otras fuentes de información que lleven a descartar el feedback recibido o a intervenir (Ifenthaler \& Yau, 2020). Para atajar estas cuestiones éticas, se deben implementar, formar, comunicar y hacer cumplir políticas institucionales claras de protección de la privacidad, al mismo tiempo que se promueve el valor de las analíticas del aprendizaje (El Alfy et al., 2019; Joksimović, Kovanovićv \& Dawson, 2019;

${ }^{2}$ Protección de datos en la UE: https://ec.europa.eu/info/law/law-topic/data-protection/data-protectioneu_en

Construir el conocimiento en la era digital: retos y reflexiones. M. Marimon-Martí, J. Cabero, L. Castañeda, C. Coll, J. M. de Oliveira, M. J. Rodríguez-Triana. 
Samuelsen, Chen \& Wasson, 2019; Ifenthaler \& Yau, 2020; Romero \& Ventura, 2020; Pargman \& McGrath, 2021). En cualquier caso, estos y nuevos problemas éticos van a requerir una revisión progresiva tanto en el corto, como en el medio y largo plazo.

\section{Conclusiones}

A continuación, se presentan las reflexiones finales de los autores de este trabajo que, sin ánimo de ser concluyentes, giran en torno a la discusión sobre cómo los desafíos que hemos identificado en las líneas anteriores reflejan la evolución de los modelos de construcción de conocimiento en la era digital entre el período 2014-2021.

\section{1 ¿Los retos identificados en 2014 son ya una realidad?}

Los retos identificados en 2014 se han hecho realidad de manera inapelable, aunque en algunos casos la pandemia y sus restricciones y urgencias han modificado el natural desarrollo de algunos de ellos, y se ha ganado un impulso nuevo que ha desencadenado niveles de colaboración para la construcción del conocimiento verdaderamente nuevos. Nadie duda ya de que el aprendizaje a lo largo de la vida es una exigencia en el mundo actual y lo va a ser cada vez más a medida que avancemos en este siglo, ya que la información está más accesible y el aprendizaje informal autodirigido es mucho más factible. La pandemia de la COVID-19 ha puesto de manifiesto, en ocasiones más por las carencias - de infraestructura, de equipamiento, de formación- detectadas que por otra cosa, que juegan un papel determinante en el aprendizaje y que lo van a jugar cada vez más, también cuando finalice la pandemia. Las ideas del seamless learning, del aprendizaje conectado, de las trayectorias de aprendizaje, de la importancia de conectar aprendizajes y experiencias de aprendizaje al margen del lugar y del momento en que se producen son omnipresentes en el discurso pedagógico actual y están en el origen de innumerables experiencias de innovación educativa en muchos países. Por otro lado, aunque las tecnologías han eliminado múltiples barreras, también se han añadido otras, siendo necesario (1) tener acceso a dispositivos móviles e Internet y (2) saber utilizar ambos con una finalidad educativa. Por último, es difícil encontrar en la actualidad una propuesta curricular, pedagógica o didáctica, o un proyecto o experiencia innovadora que no contemple la personalización del aprendizaje como uno de sus ingredientes. El uso de analíticas del aprendizaje y sistemas de recomendación ha sido de gran ayuda en esta personalización. Nadie duda actualmente de que los retos que avanzábamos en 2014 están entre los retos más importantes que enfrentan la mayoría, si no la totalidad, de los sistemas educativos nacionales y son un acicate para la búsqueda de nuevos modelos de construcción del conocimiento. Otra cosa bien distinta son los logros conseguidos en la búsqueda de respuestas a estos retos, tanto desde el punto de vista de la mejora de los sistemas educativos como de la construcción de modelos de construcción del conocimiento que den cuenta de ellos.

\section{2 ¿Hemos avanzado hacia nuevos modelos de construcción del conocimiento sensibles con los temas identificados en 2014?}

Hemos avanzado hacia nuevos modelos de construcción del conocimiento sensibles con los temas identificados en 2014, especialmente en términos tecnológicos y en generalización del uso de tecnologías por parte de la población. Esa generalización ha propiciado algunos de los avances que notamos en los retos identificados en el presente

Construir el conocimiento en la era digital: retos y reflexiones. M. Marimon-Martí, J. Cabero, L. Castañeda, C. Coll, J. M. de Oliveira, M. J. Rodríguez-Triana.

Página 18 de 32 
informe, en particular respecto a la ubicuidad de las experiencias de aprendizaje. El aprendizaje ubicuo está recibiendo cada vez más apoyo, especialmente gracias a las tecnologías móviles. Sin embargo, en términos educativos las cosas siguen más o menos en el mismo sitio. Si bien se ha avanzado en cuestiones como definiciones transnacionales de lo que es la competencia digital de los profesores y se incluyen cuestiones fundamentales para la implementación de algunos de los retos que identificamos en 2014 (huyendo de modelos estrictamente instrumentales que eran los que manejábamos en 2014), lo cierto es que la conciencia crítica de las personas que usamos las tecnologías se ha visto en parte reducida por cuestiones como la extrema polarización de los discursos, la cada vez menor diversidad en las fuentes y centros de control de Internet y las crisis sociales que hemos enfrentado (en especial la emergencia de la Covid-19) y que han estandarizado algunas soluciones tecnológicas y educativas que reducen las opciones de personalización y disuaden a los participantes de probar respuestas educativas creativas o divergentes. El aprendizaje situado y contextualizado es aún una tarea en la que seguimos avanzando, con algunas herramientas que apuntan en esta dirección (e.g., Casual Learn) pero que aún no están integradas en las prácticas de enseñanza y aprendizaje. Aún así, podemos afirmar que hemos realizado avances importantes. Por una parte, el hecho de que sean reconocidos en la actualidad de manera generalizada como retos es ya un avance. Es bien sabido que el primer paso para resolver un problema consiste en reconocerlo como tal y no ignorarlo ni minimizarlo. Por otra parte, podríamos decir que algunos de los retos seleccionados en esta edición del FIET son, al menos en parte, una reformulación de los retos formulados en 2014, una reformulación que refleja los avances realizados en la manera de plantearlos, de abordarlos y de buscar estrategias para afrontarlos. Con todo, hay que reconocer que estamos aún lejos de poder decir que los hemos superado. No obstante, el reconocimiento y la reformulación son avances que subrayan la importancia y el interés de perseverar en la tarea.

\section{3 ¿Cuáles son los nuevos retos a los que nos enfrentamos hoy?}

Los nuevos retos a los que nos enfrentamos hoy en el terreno de la investigación van en diferentes direcciones. En primer lugar, hemos de aumentar el conocimiento sobre la aplicación de las tecnologías digitales en las instituciones educativas. A pesar del aumento de la investigación en este ámbito, el conocimiento que tenemos es insuficiente, y de ahí que muchas veces la incorporación de las tecnologías no sirva para crear nuevas prácticas y entornos de comunicación. Incluso desde los informes PISA se ha señalado que la incorporación de las tecnologías no ha producido beneficios significativos y ello se debe al tipo de errores que se han cometido en la investigación realizada, que ha tenido fuertes limitaciones como la falta de fundamentación teórica de las decisiones adoptadas, el haber estado bastante cargada de una visión instrumentalista y determinista del poder transformador de las tecnologías, y el centrarse más en los instrumentos tecnológicos que en los problemas reales con los que se enfrenta el profesorado y el estudiantado en el proceso de enseñanza y en la construcción del conocimiento. Los retos son, por tanto, realizar investigaciones en el uso eeducativo de las tecnologías desde posiciones diferentes a las que tradicionalmente se han abordado, como, por ejemplo, investigar no solo el uso de las tecnologías cuando agregan un valor cuantitativo (hacer las cosas más rápido y de forma más eficaz), sino también desde una posición cualitativa (cómo alcanzar con ellas un aprendizaje auténtico y profundo y transformar los escenarios formativos) o centrarse en qué y cómo aprenden los estudiantes cuando interaccionan con las tecnologías. En este sentido, uno de los retos más importante al que nos enfrentamos

Construir el conocimiento en la era digital: retos y reflexiones. M. Marimon-Martí, J. Cabero, L. Castañeda, C. Coll, J. M. de Oliveira, M. J. Rodríguez-Triana. 
hoy, y en el que convergen de alguna manera todos los otros, es el de integrar tres dimensiones que sabemos que juegan un papel decisivo en los procesos de construcción del conocimiento, pero que todavía no hemos sido capaces de articular en el diseño de situaciones de aprendizaje ni en la elaboración de un modelo de construcción del conocimiento. Entre otras razones, porque tanto el diseño de situaciones orientadas a promover el aprendizaje como los modelos de construcción del conocimiento suelen tener en cuenta una o como máximo dos de estas dimensiones, pero hasta el momento los intentos de atender simultáneamente a las tres son muy escasos, por no decir inexistentes. Las tres dimensiones en cuestión son: presencialidad - virtualidad; sincronía - asincronía; interacción cara a cara - interacción a distancia. Cada vez más los procesos reales de construcción del conocimiento - es decir, la manera como actualmente las personas construimos conocimiento- hacen intervenir de una u otra manera estas tres dimensiones, por lo que es necesario investigar y entender cómo se combinan apoyando o interfiriendo, según los casos, en esos procesos. En este reto coinciden de alguna manera todos los otros. De ahí su importancia. Al margen de estas cuestiones, y para dar respuesta a otros retos actuales que nos permitan trabajar con nuevos paradigmas y enfoques de investigación, asumiendo una perspectiva de investigación más holística y sistémica, la investigación sobre el uso educativo de las tecnologías también debería abordar el empoderamiento y la emancipación de la población para la creación y consumo responsable de conocimiento, la justicia social (como apuesta de democratización real de la creación de conocimiento en el mundo) y la conciliación entre nuestra responsabilidad en el uso de la tecnología y su naturaleza incontrolable. Estos retos están relacionados con los cinco grandes temas tratados en el presente informe: la democratización de medios y capacidades, la articulación fluida de contextos de creación del conocimiento, la curación de contenidos, las analíticas de aprendizaje y, por supuesto, la nueva realidad inaugurada por la pandemia.

\section{4 ¿Qué nuevas consideraciones debemos tener en cuenta en los procesos de construcción de conocimiento como consecuencia de la Covid-19?}

La crisis de la Covid-19 y la respuesta generalizada a esa crisis ha tenido una serie de consecuencias que han modificado la "evolución natural" de los procesos de integración tecnológica. Esos procesos, si bien han sido gestados en condiciones de urgencia y con altas dosis de desesperación por brindar respuestas rápidas y eficientes, no han sido siempre idóneos. Corremos el riesgo evidente de que las condiciones de emergencia se perpetúen, que entendamos que las condiciones de emergencia son las ideales o que es a lo que podemos aspirar, y que eso merme la evolución real de los modelos de creación de conocimiento y de educación. La pandemia nos ha enseñado diferentes cosas: una es que muchos docentes no sabían utilizar las tecnologías para la construcción del conocimiento y las utilizaban como repositorios de objetos de aprendizaje o para dar videoconferencias presenciales; y otra es que los alumnos no son tan nativos digitales como se ha supuesto a menudo, pues las investigaciones que se han realizado sobre el uso de las tecnologías por los estudiantes señalan que normalmente las utilizan para escribir trabajos y para realizar presentaciones de clase, lo que son usos muy periféricos en los procesos de construcción del conocimiento. De ahí que una de las urgencias sea la necesidad de capacitar al profesorado para incorporar las tecnologías digitales a la práctica educativa, más allá del mero manejo instrumental de ellas, y de llevar a cabo acciones formativas con el alumnado para que desarrollen la competencia digital. La pandemia trajo también el desarrollo del aprendizaje híbrido y deberíamos seguir indagando en esa dirección. Es

Construir el conocimiento en la era digital: retos y reflexiones. M. Marimon-Martí, J. Cabero, L. Castañeda, C. Coll, J. M. de Oliveira, M. J. Rodríguez-Triana. 
importante llamar la atención sobre la falta de claridad de lo que se ha dado en llamar "aprendizaje híbrido", que se ha identificado mayoritariamente con propuestas como la alternancia de actividades en el centro escolar y en el hogar familiar con o sin acompañamiento y supervisión de los docentes, o el seguimiento desde el hogar familiar de las actividades que parte del grupo realiza en el centro escolar. La falta de claridad a este respecto tiene que ver, fundamentalmente, con lo comentado anteriormente respecto a no tener en cuenta las tres dimensiones indicadas (presencialidad - virtualidad; sincronía - asincronía; interacción cara a cara - interacción a distancia). En último término, lo que esta carencia pone de manifiesto es que las tecnologías siguen siendo utilizadas mayoritariamente en los centros educativos como instrumentos de apoyo a la enseñanza y al aprendizaje, pero no como herramientas que permiten a los aprendices enriquecer sus entornos personales de aprendizaje, dentro y fuera de los centros educativos, en solitario o con otros, e interactuando sincrónica o asincrónicamente con otros. En este sentido, en los nuevos procesos de construcción del conocimiento se ha de considerar, ineludiblemente, la relación interpersonal como su eje vertebrador. Si bien siempre la construcción de conocimiento ha evocado un compromiso social, en la actualidad, incluso en nivel de las micro-relaciones es imperativo no perder de vista que en los dos lados de la pantalla hay personas, con sentimientos, contextos, experiencias y anhelos hasta cierto punto ignorados. El reconocimiento de este hecho humaniza las relaciones que se hacen progresivamente más mediatizadas.

\section{5 ¿Cómo ha evolucionado la investigación entre 2014 y $2021 ?$}

La investigación sobre los usos educativos de las tecnologías digitales ha evolucionado fuertemente desde la primera edición del FIET en 2014 en el contexto científico nacional e internacional. Prueba de ello son las publicaciones de este ámbito tanto en revistas generalistas de educación como en revistas monográficas que han ido apareciendo progresivamente, o el volumen de las tesis realizadas sobre esta temática en los departamentos de distintas universidades. Seguramente los cambios más importantes están relacionados con nuestra visión del papel de la tecnología como consecuencia del cambio conceptual producido, de manera que se ha pasado de entender las TIC como Tecnologías de la Información y la Comunicación, a TAC, como Tecnologías para el Aprendizaje y el Conocimiento, y TEP, como Tecnologías para el Empoderamiento y la Participación. Este cambio conceptual conlleva que la investigación de vanguardia ya no "termina" en el análisis instrumental, sino que abarca temáticas más significativas que han aportado conocimiento para la incorporación de las tecnologías digitales a la enseñanza como el diseño tecno-pedagógico de las tecnologías en función de sus características sintácticas y semánticas para la realización de aprendizajes; la adaptación a las características cognitivas de los estudiantes o su utilización para el autoaprendizaje del estudiante; la relación de las tecnologías con otros elementos del currículum, desde los contenidos a las formas de evaluación; y, de forma significativa, el uso las tecnologías como configuradoras de nuevas estrategias y escenarios educativos, como por ejemplo el aprendizaje colaborativo, la clase invertida o el aprendizaje híbrido. En el caso de las analíticas de aprendizaje, la investigación durante estos últimos años ha tratado de promover la fundamentación en teorías pedagógicas, la involucración de los usuarios y un mayor énfasis en la evaluación de las propuestas, no sólo desde el punto de vista de la adopción, sino también del impacto en las prácticas educativas. Por otro lado, en los últimos años también priman las relaciones sociomateriales de las cuestiones investigadas, lo que se ha hecho cada vez más patente y generalizado en la investigación

Construir el conocimiento en la era digital: retos y reflexiones. M. Marimon-Martí, J. Cabero, L. Castañeda, C. Coll, J. M. de Oliveira, M. J. Rodríguez-Triana.

Página 21 de 32 
posterior al año 2014. Por ejemplo, preocupan los efectos sociales y conductuales que está produciendo la fuerte utilización de las tecnologías, como son la adicción y la formación de las personas para adquirir una "dieta digital" o bienestar digital. Otro fuerte avance en la investigación tiene que ver con la capacitación de los actores que utilizan las tecnologías digitales, es decir la competencia digital que docentes y discentes deben tener para una adecuada utilización educativa, social y personal de ellas. Finalmente, también se hace evidente una mayor preocupación en el ámbito de la investigación por las cuestiones de heterogeneidad en los contextos de investigación, con el fin de ofrecer una visión más global de los problemas a los que nos aproximamos. Los temas y las descripciones de los productos de investigación que se han incorporado en este documento dan ya cuenta de cómo ha evolucionado la investigación. Y es que estos productos no dejan de ser el reflejo de cómo se han ido reformulando los retos entre 2014 y el 2021 y de los avances conseguidos durante estos años, modestos pero al mismo tiempo significativos.

En resumen, y para concluir, entendemos que nuestra realidad posdigital (de Laat \& Dohn, 2019) y sus particularidades en lo que se refiere a la creación del conocimiento nos interpelan como personas interesadas en la educación de una forma profunda. No se trata solo de qué retos hay que enfrentar en adelante, sino de qué forma se han de afrontar esos retos. El escenario en el que nos encontramos exige que los debates y las soluciones que planteemos compartan una serie de rasgos fundamentales identificados en el presente trabajo:

- Deben ser inclusivos. La creación del conocimiento debe abrirse a todos y debe reflejar esa apertura tanto en sus prácticas como en sus concepciones sobre lo que significa conocer, aprender, enseñar, evaluar.

- Deben partir de y promover una realidad flexible y conectada; tanto desde un punto de vista material (flexibilidad y conexión de escenarios, contextos, herramientas), como desde un punto de vista epistémico (flexibilidad y conexión de ideas, de realidades, de subjetividades).

- Han de ser críticos. Es tiempo de revisar lo hecho hasta ahora y de profundizar en las aportaciones - positivas y negativas- de los aspectos menos instrumentales que la tecnología ha hecho al conocimiento y a las prácticas educativas, así como a nuestra respuesta - personal y colectiva- a los desafíos tecnológicos.

- Deben aprender a convivir con la incertidumbre. La realidad ha demostrado con contundencia estar más allá de las posibilidades de predicción y control de las personas. Entender el carácter emergente de la actividad -como constructosupone un cambio de paradigma a todos los niveles, incluido la creación de conocimiento y, cómo no, la educación.

- Deben poner en el centro de los objetivos educativos la capacidad de empoderar a las personas como miembros de una comunidad. La educación como creadora y garante de espacios donde las personas puedan aprender y generar conocimiento.

Somos conscientes de que nuestro análisis solo puede ser parcial, e imaginamos que quienes lean este documento seguramente identificarán otros retos y realidades relacionados con la construcción del conocimiento que interpelan igualmente de forma directa a la educación y a la tecnología. 


\section{Agradecimientos}

Los y las autoras desean agradecer a los organizadores del Forum Internacional de Educación y Tecnología (FIET), en particular a su ideóloga, la dra. Mercè Gisbert, y a su equipo del Laboratorio de Aplicaciones Telemáticas a la Educación (L@te) de la Univesitat Rovira i Virgili implicado en la organización del FIET de las ediciones 2014 y 2021, por generar espacios de reflexión crítica y propiciar la ocasión para el análisis interdisciplinar de la realidad social y educacional.

Presentación del artículo: 23 de diciembre de 2021

Fecha de aprobación: 14 de enero de 2022

Fecha de publicación: 30 de enero de 2022

Marimon-Martí, M., Cabero, J., Castañeda, L., Coll, C., de Oliveira, J.M., \& RodríguezTriana, M. J. (2022). Construir el conocimiento en la era digital: retos y reflexiones. RED. Revista Educación a Distancia, 22(69). http://dx.doi.org/10.6018/red.505661

\section{Financiación}

Este trabajo no ha recibido ninguna subvención específica de los organismos de financiación en los sectores públicos, comerciales o sin fines de lucro.

\section{Referencias}

Abdi A.A. (2015) Decolonizing Global Citizenship Education. In Abdi A.A., Shultz L., Pillay T. (eds), Decolonizing Global Citizenship Education (pp.11-26). SensePublishers. https://doi.org/10.1007/978-94-6300-277-6_2

Aditya, D.S. (2021). Embarking digital learning due to COVID-19: Are teachers ready? Journal of Technology and Science Education, 11(1), 104-116. https://doi.org/10.3926/jotse.1109

Aloizou, V., Chasiotou, T., Retalis, S., Daviotis, T., \& Koulouvaris, P. (2021). Remote learning for children with Special Education Needs in the era of COVID-19: Beyond tele-conferencing sessions. Educational Media International, 58(2), 181201. https://doi.org/10.1080/09523987.2021.1930477

Álvarez-Álvarez, C., \& García-Prieto, F. (2021). Brecha digital y nuevas formas académicas en la escuela rural española durante el confinamiento. Educar, 57(2), 397411. https://doi.org/10.5565/rev/educar.1250

Asher, N. (2010). Decolonizing Curriculum. In Malewski, E. (Ed.), Curriculum Studies Handbook-The Next Moment (pp. 393-402). Routledge \& CRC Press. https://www.routledge.com/Curriculum-Studies-Handbook---The-NextMoment/Malewski/p/book/9780415989497

Avella, J. T., Kebritchi, M., Nunn, S. G., \& Kanai, T. (2016). Learning analytics methods, benefits, and challenges in higher education: A systematic literature review. Online Learning, 20(2), 13-29. 
Banihashem, S. K., Aliabadi, K., Pourroostaei Ardakani, S., Delaver, A., \& Nili Ahmadabadi, M. (2018). Learning analytics: A systematic literature review. Interdisciplinary Journal of Virtual Learning in Medical Sciences, 9(2), 1-10. https://doi.org/10.5812/ijvlms.63024

Baran, E., \& AlZoubi, D. (2020). Affordances, challenges, and impact of open pedagogy: examining students voices. Distance Education, 41(2), 230-244. https://doi.org/10.1080/01587919.2020.1757409

Barron, B. (2006). Interest and self-sustained learning as catalysts of development: A learning ecology perspective. Human Development, 49(4), 193-224. https://doi.org/10.1159/000094368

Barron, B. (2010). Conceptualizing and tracing learning pathways over time and setting. National Society for the of Education Yearbook, 109(1), 113-127. https://doi.org/10.1177/016146811011201308

Baruch, A. F., \& Gadot, R. (2021). Social Curation Experience: Towards Authentic Learning in Preservice Teacher Training. Technology Knowledge and Learning, 26(1), 105-122. https://doi.org/10.1007/s10758-020-09449-3

Beardsley, M., Albó, L., Aragón, P., \& Hernández-Leo, D. (2021). Emergency education effects on teacher abilities and motivation to use digital technologies. British Journal of Educational Technology, 52(4), 1455-1477. https://doi.org/10.1111/bjet.13101

Burgos, C., Vázquez-Cano, E., López-Meneses, E., \& Adaos, R. (2020). DIFPRORET PROJET: Analysis of educational difficulties, proposals and challenges facing the COVID-19. IJERI: International Journal of Educational Research and Innovation, 15, 17-34. https://doi.org/10.46661/ijeri.5145

Cabero, J., \& Palacios, A. (2020). Marco europeo de competencia digital docente "DigCompEdu" y cuestionario "DigCompEdu Chek-in". Edmetic. Revista de $\begin{array}{lllll}\text { Educación } & \text { Mediática } & \text { y } & \text { TIC, } & \text { 213- }\end{array}$ 234. https://doi.org/10.21071/edmetic.v9i1.12462

Cano, S., Collazos, C., Flórez-Aristizabal, 1., Moreira, F., \& Ramírez, M. (2020). Experiencia del aprendizaje de la Educación Superior ante los cambios a nivel mundial a causa del Covid-19. Campus Virtuales, 9(2), 51-59.

Casimiro, W., Casimiro, C, Barbachán, E., \& Casimiro, J. (2020). Stress, Anguish, Anxiety and Resilience of University Teachers in the Face of Covid-19. Utopía $y$ Praxis Latinoamericana, 25(7), 453-464. https://doi.org/10.5281/zenodo.4009790

Chejara, P., Prieto, L. P., Ruiz-Calleja, A., Rodríguez-Triana, M. J., Shankar, S. K., \& Kasepalu, R. (2020, September). Quantifying collaboration quality in face-to-face classroom settings using mmla. In International Conference on Collaboration Technologies and Social Computing (pp. 159-166). Springer, Cham.

Cloonan, A. (2020). Enabling student co-curation of digital media through teachers' pedagogical research. Australian Journal of Language and Literacy, 43(3), 214-223.

Cloonan, A., Paatsch, L., \& Hutchison, K. (2019). Renewing Literature Circles: Pedagogies for Curated Multimodal Responses. Reading Teacher, 73(5), 647-656. https://doi.org/10.1002/trtr.1875 
Coll, C. (2013). La educación formal en la nueva ecología del aprendizaje: tendencias, retos y agenda de investigación. En J. L. Rodríguez Illera (Comp.), Aprendizaje y Educación en la Sociedad Digital (pp. 156-170). Universitat de Barcelona.

Coll, C., Esteban-Guitart, M., \& Iglesias, E. (2020). Aprenentatge amb sentit $i$ valor personal Estratègies, recursos i experiències de personalització educativa. Editorial Graó.

Crescenzi-Lanna, L. (2020). Multimodal Learning Analytics research with young children: A systematic review. British Journal of Educational Technology, 51(5), 1485-1504. https://doi.org/10.1111/bjet.12959

Darling-Hammond, L., Flook, L., Cook-Harvey, Ch., Barron, B., \& Osher, D. (2020) Implications for educational practice of the science of learning and development. Applied Developmental Science, 24(2), 97-140. https://doi.org/10.1080/10888691.2018.1537791

de Laat, M., \& Dohn, N. B. (2019). Is Networked Learning Postdigital Education? Postdigital Science and Education, 1(1), 17-20. https://doi.org/10.1007/s42438-01900034-1

de Oliveira, J. M., Henriksen, D., Castañeda, L., Marimon, M., Barberà, E., Monereo, C., Coll, C., Mahiri, J., \& Mishra, P. (2015). The educational landscape of the digital age: Communication practices pushing (us) forward. RUSC. Universities and Knowledge Society Journal, 12(2), 14-29. http://dx.doi.org/10.7238/rusc.v12i2.2440

de Silva, L. M. H., Rodríguez-Triana, M. J., Chounta, I. A., \& Pishtari, G. (2021). An overview of analytics for curriculum understanding and optimization in Higher Education. Companion Proceedings 11th International Conference on Learning Analytics \& Knowledge (LAK21): 11th International Conference on Learning Analytics \& Knowledge (LAK21). Society for Learning Analytics Research (SoLAR).

Di Mitri, D., Schneider, J., Specht, M., \& Drachsler, H. (2019). Detecting mistakes in CPR training with multimodal data and neural networks. Sensors, 19(14), 3099. https://doi.org/10.3390/s19143099

Du, X., Yang, J., Shelton, B. E., Hung, J. L., \& Zhang, M. (2021). A systematic metareview and analysis of learning analytics research. Behaviour \& Information Technology, 40(1), 49-62. https://doi.org/10.1080/0144929X.2019.1669712

Ebersold, S. (2021). The Accessibility Imperative: Outlines and Implications. In S. Ebersold (Ed.), Accessibility or Reinventing Education (pp. 1-21). John Wiley \& Sons, Ltd. https://doi.org/10.1002/9781119817956.ch1

Echeverria, V., Martinez-Maldonado, R., Power, T., Hayes, C., \& Shum, S. B. (2018, June). Where is the nurse? Towards automatically visualising meaningful team movement in healthcare education. In International conference on artificial intelligence in education (pp. 74-78). Springer, Cham.

El Alfy, S., Marx Gómez, J., \& Dani, A. (2019). Exploring the benefits and challenges of learning analytics in higher education institutions: A systematic literature review. Information Discovery and Delivery, 47(1), 25-34. https://doi.org/10.1108/IDD-062018-0018 
Ellis, V., Steadman, S., \& Mao, Q. (2020). Come to a screeching halt: Can change in teacher education during the COVID-19 pandemic be seen as innovation? European Journal of Teacher Education, 43(4), 559-572. https://doi.org/10.1080/02619768.2020.1821186

Engzell, P., Frey, A., \& Verhagen, M. D. (2021). Learning loss due to school closures during the COVID-19 pandemic. Proceedings of the National Academy of Sciences, 118(17), e2022376118. https://doi.org/10.1073/pnas.2022376118

Esteban-Guitart, M. (2021). L'articulació d'espais i temps educatius: les trajectòries personals d'aprenentatge com a focus de l'acció educativa. In C. Coll \& B. Albaigés (Directors), L'estat de l'educació a Catalunya. Anuari 2020 (pp. 486-523). Fundació Bofill.

Esteban-Guitart, M., Coll, C., \& Penuel, W. R. (2018). Learning across settings and time in the Digital age. Digital Education Review, 33, 1-16. https://doi.org/10.1344/der.2018.33.\%25p

Esteve-Mon, F., Castañeda, L., \& Adell, J. (2018). Un modelo holístico de competencia docente para el mundo digital. Revista Interuniversitaria de Formación del Profesorado, 32(1), 105-116.

Ewing, L., \& Cooper, H. B. (2021). Technology-enabled remote learning during Covid19: perspectives of Australian teachers, students and parents. Technology, Pedagogy and Education, 30(1), 41-57. https://doi.org/10.1080/1475939X.2020.1868562

Goodyear, P., Carvalho, L., Yeoman, P., Castañeda, L., \& Adell, J. (2021). Una herramienta tangible para facilitar procesos de diseño y análisis didáctico: Traducción y adaptación transcultural del Toolkit ACAD. Píxel-Bit. Revista de Medios y Educación, 60, 7-28. https://doi.org/10.12795/pixelbit.84457

Hakimi, L., Eynon, R., \& Murphy, V. A. (2021). The Ethics of Using Digital Trace Data in Education: A Thematic Review of the Research Landscape. Review of Educational Research, 91(5), 671-717. https://doi.org/10.3102/00346543211020116

Hu, S. H., Torphy, K. T., Evert, K., \& Lane, J. L. (2020). From Cloud to Classroom: Mathematics Teachers' Planning and Enactment of Resources Accessed Within Virtual Spaces. Teachers College Record, 122(6), Article 060306.

Hu, S. H., Torphy, K. T., \& Opperman, A. (2019). Culturally Relevant Curriculum Materials in the Age of Social Media and Curation. Teachers College Record, 121(14), Article 140309. https://doi.org/10.1177/016146811912101409

Ifenthaler, D., \& Yau, J. Y. K. (2020). Utilising learning analytics to support study success in higher education: a systematic review. Educational Technology Research and Development, 68(4), 1961-1990. https://doi.org/10.1007/s11423-020-09788-z

Inciarte, A., Paredes-Chacín, A. J., \& Zambrano, L. M. (2020). Docencia y tecnologías en tiempos de pandemia covid-19. Utopía y Praxis Latinoamericana, 25(1), 195-215.

Ito, M., Gutiérrez, K., Livingstone, S., Penuel, B., Rhodes, J., Salen, K., Schor, J., SeftonGreen, J., \& Watkins, S. (2013). Connected Learning: An Agenda for Research and Design. Digital Media and Learning Research Hub. https://clalliance.org/publications/

Ito, M., Arum, R., Conley, D., Gutiérrez, K., Kirshner, B., Livingstone, S., Michalchik, V., Penuel, W., Peppler, K., Pinkard, N., Rhodes, J., Salen Tekinbaş, K., Schor, J., 
JSefton-Green, J., \& Watkins, S. C. (2020). The Connected Learning Research Network: Reflections on a Decade of Engaged Scholarship. Digital Media and Learning Research Hub. https://clalliance.org/publications/

Joksimović, S., Kovanović, V., \& Dawson, S. (2019). The journey of learning analytics. HERDSA Review of Higher Education, 6, 27-63.

Kidd, W., \& Murray, J. (2020). The Covid-19 pandemic and its effects on teacher education in England: how teacher educators moved practicum learning online. European Journal of Teacher Education, 43(4), 542-558. https://doi.org/10.1080/02619768.2020.1820480

Kress, G. (2003). Literacy in the new media age. Routledge.

Kress, G., \& Selander, S. (2012). Multimodal design, learning and cultures of recognition. The Internet and Higher Education, 15(4), 265-268. https://doi.org/10.1016/j.iheduc.2011.12.003

Kuhfeld, M., Soland, J., Tarasawa, B., Johnson, A., Ruzek, E., \& Liu, J. (2020). Projecting the Potential Impact of COVID-19 School Closures on Academic Achievement. Educational Researcher, 49(8), 549-565. https://doi.org/10.3102/0013189X20965918

Lambert, S., \& Czerniewicz, L. (2020). Approaches to Open Education and Social Justice Research. Journal of Interactive Media in Education, 2020(1), 1. https://doi.org/10.5334/jime.584

Larrabee Sønderlund, A., Hughes, E., \& Smith, J. (2019). The efficacy of learning analytics interventions in higher education: A systematic review. British Journal of Educational Technology, 50(5), 2594-2618. https://doi.org/10.1111/bjet.12720

Liu, Y. Q., Torphy, K. T., Hu, S. H., Tang, J. L., \& Chen, Z. X. (2020). Examining the Virtual Diffusion of Educational Resources Across Teachers' Social Networks Over Time. Teachers College Record, 122(6), Article 060305. https://doi.org/10.1177/016146812012200605

Llopart, M., \& Esteban-Guitart, M. (2018). Funds of knowledge in 21st century societies: Inclusive educational practices for under-represented students. A literature review. Journal of Curriculum Studies, 50(2), 145-161. https://doi.org/10.1080/00220272.2016.1247913

Longino, H. E. (2017). Feminist Epistemology. In J. Greco, \& E. Sosa (Eds), The Blackwell Guide to Epistemology (pp. 325-353). John Wiley \& Sons, Ltd. https://doi.org/10.1002/9781405164863.ch14

Maile, R., Mena, J., \& Feinauer, E. (2020). Faculty readiness for online crisis teaching: transitioning to online teaching during the COVID-19 pandemic. European Journal of Teacher Education, 43(4), 523-541. https://doi.org/10.1080/02619768.2020.1815702

Mangaroska, K., \& Giannakos, M. (2018). Learning analytics for learning design: A systematic literature review of analytics-driven design to enhance learning. IEEE Transactions on Learning Technologies, 12(4), 516-534. https://doi.org/10.1109/TLT.2018.2868673

Marimon, M., Barberà, E., Coll, C., \& Monereo, C. (2016). Nous models de construcció de coneixement. In M. Gisbert \& J. González (Eds.), Nous escenaris d'aprenentatge 
des d'una visió transformadora. New learning environments from a transformative perspective (pp. 57-78). Wolters Kluver.

Martín, I. M., \& Artiaga, G. R. (2017). Des-patriarcalizar y Des-colonizar la Educación. Experiencias para una Formación Feminista del Profesorado. Revista Internacional de Educación para la Justicia Social, 6(2). https://doi.org/10.15366/riejs2017.6.2.005

Morocho, M. E. (2021). El Aseguramiento de la Calidad de la Educación Superior en Latinoamérica y el Caribe, en tiempos del COVID-19: Visión de las Instituciones de Educación Superior. Instituto Latinoamericano y del Caribe de Calidad en Educación Superior a Distancia (CALED-Ecuador) Universidad Técnica Particular de Loja (UTPL-Ecuador). http://www.caled-ead.org/sites/default/files/Libro-InstitucionesES.pdf

Morozov, V., \& Pavlova, E. (2018). Indigeneity and subaltern subjectivity in decolonial discourses: A comparative study of Bolivia and Russia. Journal of International Relations and Development, 21(3), 689-716. https://doi.org/10.1057/s41268-0160076-7

Navarro-Espinosa, J. A., Vaquero-Abellán M., Perea-Moreno A. J., Pedrós-Pérez G., Aparicio-Martínez P., \& Martínez-Jiménez M. P. (2021). The Higher Education Sustainability before and during the COVID-19 Pandemic: A Spanish and Ecuadorian Case. Sustainability, 13(11), 6363. https://doi.org/10.3390/su13116363.

Networked Learning Editorial Collective (NLEC). (2021). Networked Learning: Inviting Redefinition. Postdigital Science and Education, 3, 312-325. https://doi.org/10.1007/s42438-020-00167-8

Nieto, D. (2018). Citizenship education discourses in Latin America: Multilateral institutions and the decolonial challenge. Compare: A Journal of Comparative and International Education, 48(3), 432-450. https://doi.org/10.1080/03057925.2017.1408399

Ochoa, X., \& Dominguez, F. (2020). Controlled evaluation of a multimodal system to improve oral presentation skills in a real learning setting. British Journal of Educational Technology, 51(5), 1615-1630. https://doi.org/10.1111/bjet.12987

Papamitsiou, Z. K., \& Economides, A. A. (2014). Learning analytics and educational data mining in practice: A systematic literature review of empirical evidence. Educational Technology \& Society, 17(4), 49-64.

Pargman, T. C., \& McGrath, C. (2021). Mapping the Ethics of Learning Analytics in Higher Education: A Systematic Literature Review of Empirical Research. Journal of Learning Analytics, 8(2), 123-139. https://doi.org/10.18608/jla.2021.1

Parmigiani, D., Benigno, V., Giusto, M., Silvaggio, Ch., \& Sperandio, S. (2021). Einclusion: online special education in Italy during the Covid-19 pandemic. Technology, Pedagogy and Education, 30(1), 111-124. https://doi.org/10.1080/1475939X.2020.1856714

Peters, M. A., Besley, T., \& Jandrić, P. (2018). Postdigital Knowledge Cultures and Their Politics. ECNU Review of Education, 1(2), 23-43. https://doi.org/10.30926/ecnuroe2018010202 
Potter, J. (2011). New literacies, new practices and learner research: Across the semipermeable membrane between home and school. Lifelong Learning in Europe, 16(3), 174-181.

Rodríguez-Triana, M. J., Prieto, L. P., Martínez-Monés, A., Asensio-Pérez, J. I., \& Dimitriadis, Y. (2018, March). The teacher in the loop: Customizing multimodal learning analytics for blended learning. In Proceedings of the 8th international conference on learning analytics and knowledge (pp. 417-426).

Roll, I., \& Winne, P. H. (2015). Understanding, evaluating, and supporting self-regulated learning using learning analytics. Journal of Learning Analytics, 2(1), 7-12. https://doi.org/10.18608/jla.2015.21.2

Romero, C., \& Ventura, S. (2020). Educational data mining and learning analytics: An updated survey. Wiley Interdisciplinary Reviews: Data Mining and Knowledge Discovery, 10(3), e1355. https://doi.org/10.1002/widm.1355

Ruiz-Calleja, A., Prieto, L.P., Ley, T., Rodríguez-Triana, M.J., Dennerlein, S. (2021). Learning Analytics for Professional and Workplace Learning: A Literature Review. IEEE Transactions on Learning Technologies, 14(3), 353-366. https://doi.org/10.1109/TLT.2021.3092219

Ryan, T. (2021). Designing video feedback to support the socioemotional aspects of online learning. Education Tech Research Dev, 69, 137-140 https://doi.org/10.1007/s11423-020-09918-7

Samuelsen, J., Chen, W., \& Wasson, B. (2019). Integrating multiple data sources for learning analytics - review of literature. Research and Practice in Technology Enhanced Learning, 14(1), 1-20. https://doi.org/10.1186/s41039-019-0105-4

Santos, B. de S., \& Martins, B. S. (2021). The Pluriverse of Human Rights: The Diversity of Struggles for Dignity: The Diversity of Struggles for Dignity. Routledge. Taylor \& Francis Group.

Santos, B. de S. (2018). The End of the Cognitive Empire: The Coming of Age of Epistemologies of the South. Duke University Press.

Sawyer, K. (Ed.) (2014). The Cambridge Handbook of the Learning Sciences. 2nd Edition. Cambridge University Press.

Sawyer, A. G., Dredger, K., Myers, J., Barnes, S., Wilson, R., Sullivan, J., \& Sawyer, D. (2019). Developing Teachers as Critical Curators: Investigating Elementary Preservice Teachers' Inspirations for Lesson Planning. Journal of Teacher Education, 71(5), 518-536. https://doi.org/10.1177/0022487119879894

Schleicher, A. (2020). The impact of covid-19 on education insights from education at a glance 2020. OECD Secretariat for the European Union. https://www.oecd.org/education/the-impact-of-covid-19-on-education-insightseducation-at-a-glance-2020.pdf

Schwendimann, B. A., Rodriguez-Triana, M. J., Vozniuk, A., Prieto, L. P., Boroujeni, M. S., Holzer, A., Gillet, D., \& Dillenbourg, P. (2017). Perceiving learning at a glance: A systematic literature review of learning dashboard research. IEEE Transactions on Learning Technologies, 10(1), 30-41. https://doi.org/10.1109/TLT.2016.2599522 
Scully, D., Lehane, P., \& Scully, C. (2021). It is no longer scary': digital learning before and during the Covid-19 pandemic in Irish secondary schools. Technology, Pedagogy and Education, 30(1), 159-181. https://doi.org/10.1080/1475939X.2020.1854844

Sharma, K., Niforatos, E., Giannakos, M., \& Kostakos, V. (2020). Assessing cognitive performance using physiological and facial features: Generalizing across contexts. Proceedings of the ACM on Interactive, Mobile, Wearable and Ubiquitous Technologies, 4(3), 1-41. https://doi.org/10.1145/3411811

Torphy, K., Liu, Y. Q., Hu, S. H., \& Chen, Z. X. (2020). Sources of Professional Support: Patterns of Teachers' Curation of Instructional Resources in Social Media. American Journal of Education, 127(1), 13-47. https://doi.org/10.1086/711008

Trujillo Sáez, F. (2021). The school year 2020-2021 in Spain during the pandemic. Publications Office of the European Union, Luxembourg. https://doi.org/10.2760/729245

Valle, N., Antonenko, P., Dawson, K., \& Huggins-Manley, A. C. (2021). Staying on target: A systematic literature review on learner-facing learning analytics dashboards. British Journal of Educational Technology, 52(4), 1724-1748. https://doi.org/10.1111/bjet.13089

Viberg, O., Hatakka, M., Bälter, O., \& Mavroudi, A. (2018). The current landscape of learning analytics in higher education. Computers in Human Behavior, 89, 98-110. https://doi.org/10.1016/j.chb.2018.07.027

Vieira, C., Parsons, P., \& Byrd, V. (2018). Visual learning analytics of educational data: A systematic literature review and research agenda. Computers \& Education, 122, 119-135. https://doi.org/10.1016/j.compedu.2018.03.018

Wong, B. T. M., \& Li, K. C. (2020). A review of learning analytics intervention in higher education (2011-2018). Journal of Computers in Education, 7(1), 7-28. https://doi.org/10.1007/s40692-019-00143-7

Yates, A., Starkey, L., Egerton, B., \& Flueggen, F. (2021). High school students' experience of online learning during Covid-19: the influence of technology and pedagogy. Technology, Pedagogy and Education, 30(1), 59-73. https://doi.org/10.1080/1475939X.2020.1854337

Yedaide, M. M., \& Porta, L.G. (2020). Decolonial pedagogies, narrative research and the epistemological whereabouts of teacher education in a global south. Journal for Educators, Teachers and Trainers, 10(1), 217 - 228. http://hdl.handle.net/10481/60028

Zawacki-Richter, O., Marín, V. I., Bond, M., \& Gouverneur, F. (2019). Systematic review of research on artificial intelligence applications in higher education - where are the educators? International Journal of Educational Technology in Higher Education, 16(1), 39. https://doi.org/10.1186/s41239-019-0171-0 


\section{Anexo. Glosario de los principales términos utilizados en el texto}

Accesibilidad. Condición mediante la cual un entorno o producto es susceptible de ser utilizado por cualquier persona, sean cuales sean sus condiciones físicas, psíquicas o sensoriales.

Analíticas del aprendizaje. Hace referencia tanto al campo académico como a las tecnologías destinadas a la medición, recopilación, análisis y presentación de datos sobre los alumnos y sus contextos, con el fin de comprender y optimizar el aprendizaje y los entornos en los que se produce.

Aprendizaje autónomo. Proceso de aprendizaje en el que la persona aprende por sí misma, dirigiendo y autoorganizando su proceso de aprendizaje. Normalmente se apoya en diferentes recursos y medios de enseñanza, tanto analógicos como digitales.

Aprendizaje con sentido. Un aprendizaje tiene sentido y valor personal para el aprendiz cuando le sirve para: (i) conocerse mejor, ayudándole a resignificar retrospectivamente una experiencia pasada o un aprendizaje previo; (ii) conocer y comprender mejor la realidad en la que está inmerso y a actuar en y sobre ella; (iii) proyectarse hacia el futuro elaborando planes de acción en los ámbitos personal, profesional o social. Los aprendizajes con sentido y valor personal son un ingrediente fundamental del proceso de construcción de la identidad.

Competencias digitales. Uso creativo, crítico y seguro de las TIC para alcanzar los objetivos relacionados con el trabajo, la empleabilidad, el aprendizaje, el tiempo libre, la inclusión y participación en la sociedad.

Contextos situados de aprendizaje. Contexto analógico, virtual y sociocultural donde se ubica el proceso de enseñanza y aprendizaje y lo concreta.

Curación de contenidos. Práctica de identificar, catalogar y compartir recursos multimodales con objetivos comunicativos o de aprendizaje específicos. Influye en el proceso de construcción y representación identitario, a la vez que se ve influida por la identidad.

Descolonización de los discursos. Proceso que va más allá de la independencia política de los territorios y se refiere a la independencia intelectual de los discursos asociados a determinados grupos sociales en relación con los discursos histórica y culturalmente dominantes.

Desigualdades ante el aprendizaje. Los contextos de actividad por los que transitan y en los que participan las personas difieren considerablemente entre sí en lo que concierne a la amplitud y riqueza de las oportunidades y recursos para aprender que ponen a disposición de las personas que acceden a ellos. En la medida en que el acceso y la participación a unos u otros de estos contextos de actividad está fuertemente asociado a variables de naturaleza socioeconómica y cultural, se generan fuertes desigualdades entre las personas en las oportunidades y recursos para aprender que tienen a su alcance. 
Diversidad. Característica en la producción de conocimiento, no solo por la participación en esa creación de diferentes culturas, grupos sociales y personas con perspectivas heterogéneas, sino por la variedad en formas de producción, distribución, almacenamiento y consumo, en múltiples organizaciones y sociedades.

Educación distribuida e interconectada. Visión de la educación acorde con la nueva ecología del aprendizaje, una de cuyas características principales es la multiplicación y diversificación de los contextos de actividad, a menudo de la mano de las tecnologías digitales de la información y la comunicación, que ofrecen oportunidades y recursos para aprender. En un modelo de educación distribuida e interconectada el foco de la acción educativa son las trayectorias personales de aprendizaje y su finalidad es ayudar a los aprendices a construir trayectorias ricas y potentes que les permitan seguir aprendiendo en una amplia gama de situaciones y condiciones.

Inteligencia artificial. Rama de la ciencia que produce y estudia las técnicas de procesamiento de información y computación cuyo objetivo es permitir que las computadoras realicen tareas mediante la simulación de comportamientos humanos inteligentes, como inferencias, análisis y toma de decisiones.

Minería de datos educativos. Disciplina preocupada por desarrollar métodos para explorar los datos, especialmente a gran escala, que provienen de entornos educativos y utilizar esos métodos para comprender mejor a los estudiantes y los entornos en los que aprenden.

Prácticas innovadoras. Conjunto de actividades y estrategias educativas que buscan cambios cualitativos en una situación específica de enseñanza, resolver un problema identificado en el aprendizaje, en la comunicación, en la convivencia o en la gestión escolar. Pueden apoyarse en la incorporación de tecnologías digitales.

Recursos Flexibles. Características de un recurso digital que facilita su utilización en una diversidad de contextos, para una diversidad de acciones educativas y respetando una diversidad de características y condiciones de la persona.

Recursos multimodales. Objetos semióticos que combinan más de un lenguaje o formato en su composición (visual -estático y/o dinámico-, sonoro, verbal multimedia, hipertextuales, hipermedia, etc.).

Trayectorias personales de aprendizaje. Conjunto de experiencias de aprendizaje vividas y de aprendizajes realizados por las personas a lo largo de la vida en su tránsito por diferentes contextos de actividad, propios de la educación formal y ajenos a ella, que ofrecen oportunidades y recursos para aprender a quienes acceden y participan en ellos. 